\title{
Green's function retrieval from reflection data, in absence of a receiver at the virtual source position
}

\author{
Kees Wapenaar, a) Jan Thorbecke, and Joost van der Neut \\ Department of Geoscience and Engineering, Delft University of Technology, 2600 GA Delft, The Netherlands \\ Filippo Broggini ${ }^{\text {b) }}$ \\ Center for Wave Phenomena, Colorado School of Mines, Golden, Colorado 80401 \\ Evert Slob \\ Department of Geoscience and Engineering, Delft University of Technology, 2600 GA Delft, The Netherlands \\ Roel Snieder \\ Center for Wave Phenomena, Colorado School of Mines, Golden, Colorado 80401
}

(Received 30 June 2013; revised 26 November 2013; accepted 6 March 2014)

\begin{abstract}
The methodology of Green's function retrieval by cross-correlation has led to many interesting applications for passive and controlled-source acoustic measurements. In all applications, a virtual source is created at the position of a receiver. Here a method is discussed for Green's function retrieval from controlled-source reflection data, which circumvents the requirement of having an actual receiver at the position of the virtual source. The method requires, apart from the reflection data, an estimate of the direct arrival of the Green's function. A single-sided three-dimensional (3D) Marchenko equation underlies the method. This equation relates the reflection response, measured at one side of the medium, to the scattering coda of a so-called focusing function. By iteratively solving the 3D Marchenko equation, this scattering coda is retrieved from the reflection response. Once the scattering coda has been resolved, the Green's function (including all multiple scattering) can be constructed from the reflection response and the focusing function. The proposed methodology has interesting applications in acoustic imaging, properly accounting for internal multiple scattering. (C) 2014 Acoustical Society of America. [http://dx.doi.org/10.1121/1.4869083]
\end{abstract}

PACS number(s): 43.60.Tj, 43.40.Ph, 43.30.Gv, 43.60.Ac [JDM]

Pages: $2847-2861$

\section{INTRODUCTION}

The one-dimensional (1D) Marchenko equation is an exact integral equation which relates the reflection response, measured at one side of a medium, to a field inside that medium. ${ }^{1}$ It finds its most important applications in 1D inverse scattering problems. ${ }^{2-4}$

In a series of seminal papers, $\operatorname{Rose}^{5-7}$ discusses the link between 1D autofocusing and the Marchenko equation. Autofocusing is a process that uses the single-sided reflection response to derive a focusing wave field, which, when emitted from one side into the medium, focuses at one particular point in that medium. The work of Rose shows that the focusing field is equal to a delta pulse, minus the timereversed solution of the 1D Marchenko equation. Hence, this links autofocusing to inverse scattering.

Broggini and Snieder ${ }^{8}$ link the autofocusing method of Rose to Green's function retrieval in 1D media. They show that the superposition of the time-reversal of the focusing wave field and its reflection response yields the 1D Green's function of the medium (including all multiple scattering), with its source at the position of the focal point inside the medium. Since the focusing wave field is obtained by

\footnotetext{
a) Author to whom correspondence should be addressed. Electronic mail: C.P.A.Wapenaar@TUDelft.NL

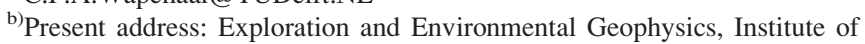
Geophysics, ETH Zürich, Zürich, Switzerland.
}

solving the Marchenko equation, the Green's function is thus retrieved entirely from the single-sided reflection response of the medium.

The latter link is remarkable because it implies that a virtual source can be created at any position in the medium, without requiring the presence of actual receivers inside the medium. This is different from the recent methodology of Green's function retrieval by cross-correlation, which has been developed for ambient noise recordings ${ }^{9-12}$ as well as for controlled-source data. ${ }^{13-16}$ In these methods, the Green's function between two receivers is obtained by cross-correlating wave fields measured at these two receivers. Hence, with these methods, one of the receivers is turned into a virtual source, whereas the other receiver measures the response to this virtual source. In seismology these methods are also called "seismic interferometry." The method of Broggini and Snieder, ${ }^{8}$ by which the Green's function is retrieved without requiring a receiver at the position of the virtual source, is therefore also called "beyond seismic interferometry." 17 The aim of this paper is to generalize the Marchenko equation and its application in Green's function retrieval to three dimensions.

The Marchenko equation has previously been extended to a three-dimensional (3D) equation, which is known as the Newton-Marchenko equation. ${ }^{18,19}$ However, solving the Newton-Marchenko equation requires measurements all around the scattering object (i.e., reflection and transmission measurements). In Secs. III-VI of this paper, a 3D Marchenko equation is derived which relates the single-sided reflection 
response of a 3D medium to a field inside that medium, similar to the 1D Marchenko equation. In Secs. VII and VIII it is shown how an iterative solution method for the single-sided 3D Marchenko equation leads to the 3D Green's function (including all multiple scattering), with its source point inside the medium where no actual receiver is present. Knowing this Green's function is the first step toward imaging the medium, properly accounting for multiple reflections. ${ }^{20,21}$ Before starting the derivations, Sec. II illustrates the classical approach to Green's function retrieval from reflection data with a numerical example.

\section{GREEN'S FUNCTION RETRIEVAL FROM \\ REFLECTION DATA: THE CLASSICAL APPROACH}

The classical approach to retrieving the 3D Green's function from reflection data ignores multiple scattering. ${ }^{22,23}$ This is illustrated here with a numerical example, with the aim to indicate the limitations and to explain the main purpose of the proposed Green's function retrieval approach.

Figure 1(a) shows a layered model, with curved interfaces between the layers, and different propagation velocities and mass densities within the layers. A source, indicated by the star at the surface, emits a wave field into the medium which, after propagation and scattering inside the medium, is detected by 451 receivers at the surface, with an inter- receiver distance of $10 \mathrm{~m}$, indicated by the triangles at the surface (only every 15 th receiver is shown). The numerically modeled reflection response is shown in gray-level display in Fig. 1(b). It has been convolved with a source function with a central frequency of $20 \mathrm{~Hz}$. Note that this response contains triplicated arrivals and internal multiple reflections [the response is displayed with a small time gain of $\exp (0.1 * t)$ to emphasize these multiples]. Reflection responses like the one in Fig. 1(b) are modeled for a total of 451 source positions at the surface, with an interval of $10 \mathrm{~m}$. The aim of this numerical experiment is to retrieve the Green's function between a virtual source inside the medium (indicated by the star inside the medium) and the receivers at the surface. Figure 1(c) shows the directly modeled Green's function, which will serve as a reference [here a time gain of $\exp (1.2 * t)$ has been applied].

Suppose that a smooth model of the medium is available, as shown in Fig. 1(d). Then an estimate of the direct arrival of the Green's function can be obtained by numerically modeling the response to the source in the smooth model. The result is shown in Fig. 1(e). This direct Green's function is now used to focus the sources of the reflection responses to the position of the virtual source inside the medium. This involves cross-correlation of the reflection responses with the direct Green's function and integration along the sources at the surface. The result approximates the response to a (a)

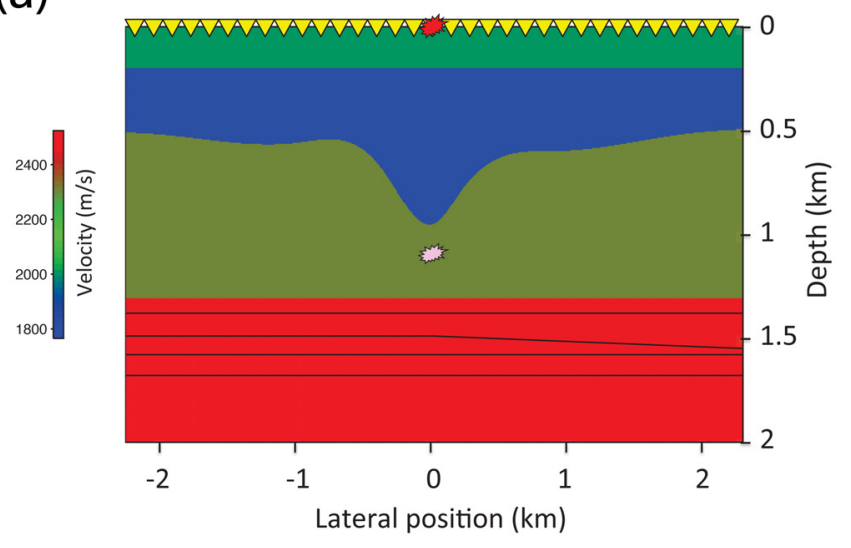

(b)

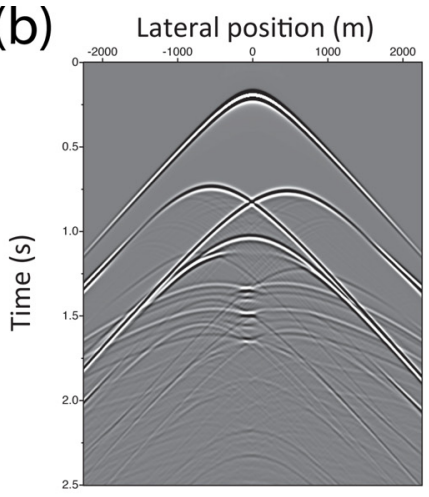

(c)

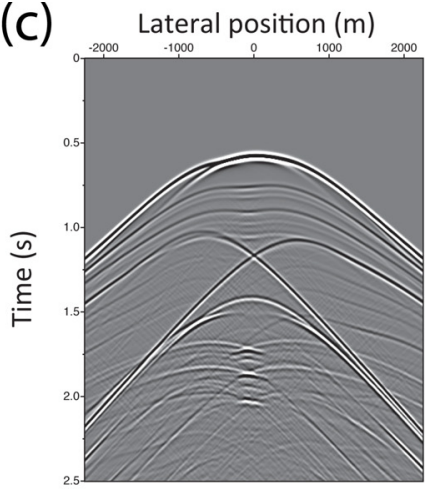

(d)

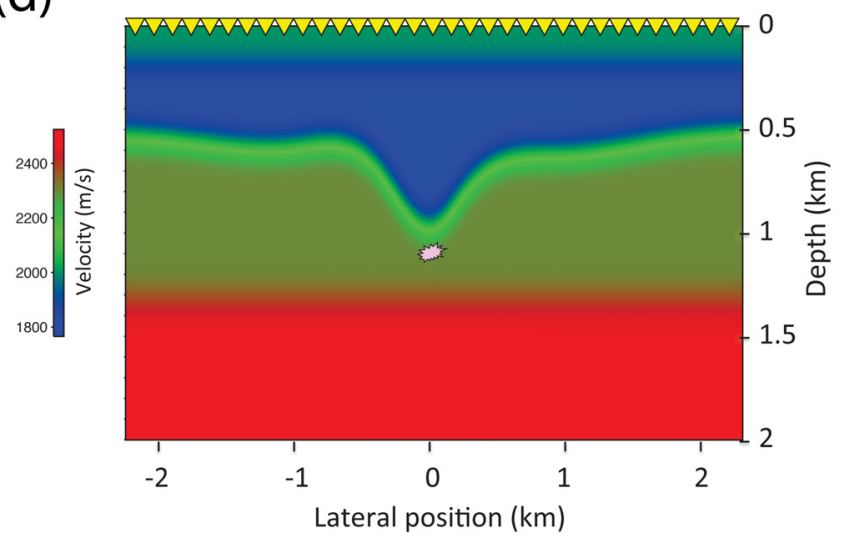

(e)

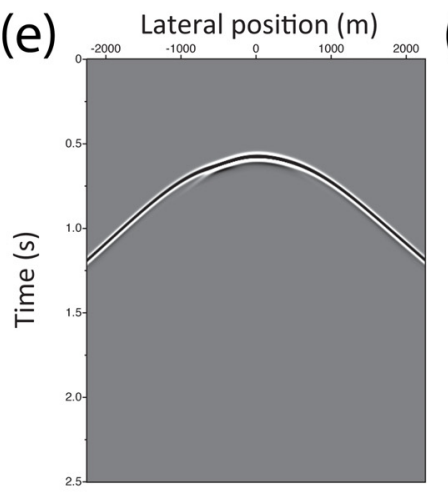

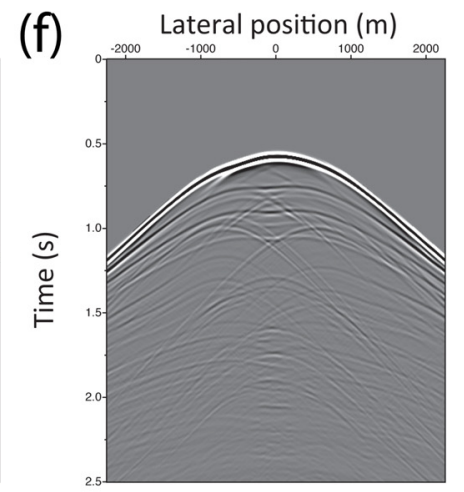

FIG. 1. (Color online) (a) Propagation velocity model. (b) Reflection response of the model in (a). The source is indicated by the star at the surface. (c) Green's function in the model in (a). The source is indicated by the star inside the medium. (d) Smoothed version of the propagation velocity model. (e) Direct arrival of the Green's function in the smoothed model of (d). (f) Green's function, retrieved by the classical approach. 
downward radiating source at the virtual-source position. The direct Green's function itself approximates the response to an upward radiating source at the virtual-source position. Hence, the superposition of these responses approximates the total Green's function between the virtual source inside the medium and the receivers at the surface. This retrieved Green's function is shown in Fig. 1(f) (after muting acausal events before the first arrival). The same result is represented by the thin black lines in Fig. 2(a) [only every 15th trace is shown, corresponding with the receivers indicated by the triangles in Fig. 1(a); a time gain of $\exp (1.9 * t)$ has been applied]. As a reference, the thick gray lines represent the directly modeled Green's function. The central trace of Fig. 2(a) is shown with more detail in Fig. 2(b). Note that the match between the retrieved and the directly modeled Green's function is reasonably accurate for the early arrivals. These early arrivals correspond to the direct arrival and the primary reflections from the reflectors below the virtual source in Fig. 1(a). The later arrivals, corresponding to the multiple reflections, are not correctly retrieved.

The theory discussed in Secs. III-VIII leads to an improved methodology for Green's function retrieval, with the aim to recover primary as well as multiple reflections. The new methodology, called the Marchenko approach to Green's function retrieval, uses the same information as the classical approach, i.e., reflection data at the surface and an

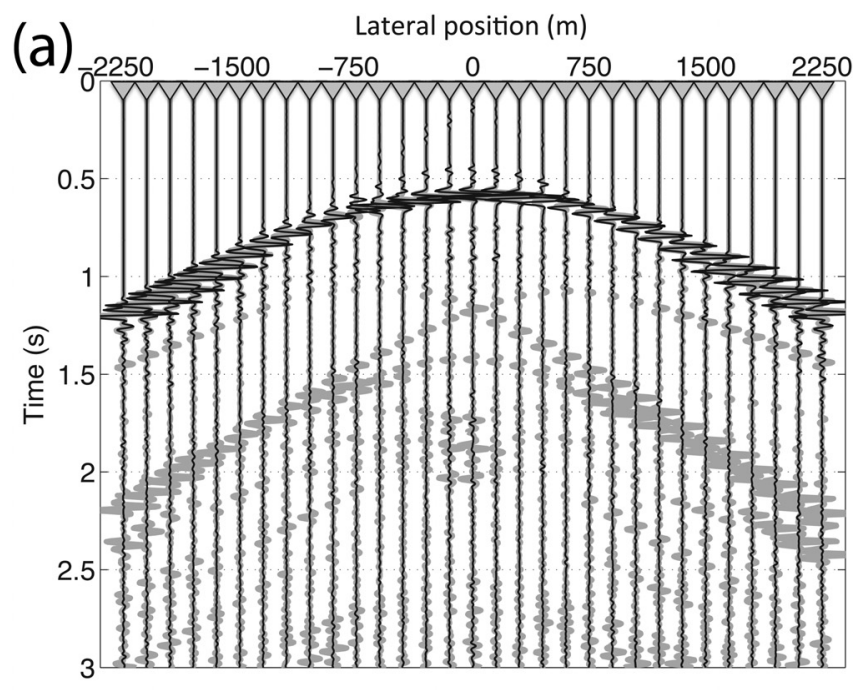

(b)

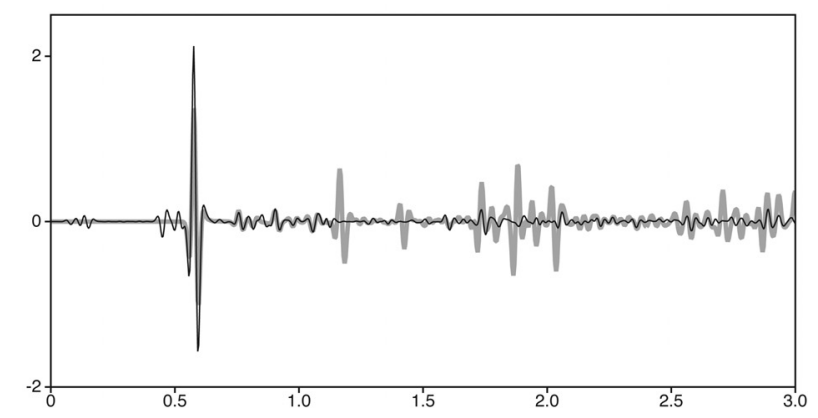

FIG. 2. Retrieved Green's function, using the classical approach (thin black lines), overlain on the directly modeled Green's function (thick gray lines). (a) Green's function at all receivers in Fig. 1(a). (b) Green's function at the central receiver in Fig. 1(a). estimate of the direct arrival of the Green's function. The Marchenko approach retrieves the multiples in the Green's function from the reflection data in an iterative way, but it does not improve the travel time of the direct arrival, thus leaving a small uncertainty in the position of the virtual source. If one seeks to know the Green's function for a given source point inside the medium, one needs to prescribe somehow where that point is located. Ideally one would like to give the coordinates, but the direct waves depend only on the location of this point through the travel time of these waves. This is the reason why one must prescribe the travel time of the first arriving waves: It is through this travel time that the virtual source point is defined. The problem of estimating the travel time of the direct arrival and pinpointing the position of the virtual source is in essence the same as for the classical approach. This is a separate issue, beyond the scope of this paper.

\section{RECIPROCITY THEOREMS FOR ONE-WAY WAVE FIELDS}

Reciprocity theorems for one-way wave fields form the basis for deriving a specific form of Green's function representation (Sec. V) which, in turn, underlies the single-sided 3D Marchenko equation (Sec. VI). This section starts with the basics of flux-normalized one-way wave fields and reviews reciprocity relations between one-way wave fields in two different states.

An acoustic wave field in the space-time domain is represented by the acoustic pressure $p(\mathbf{x}, t)$ and particle velocity $v_{m}(\mathbf{x}, t)$, where $\mathbf{x}$ is the Cartesian spatial coordinate vector, $t$ denotes time and subscript $m$ takes on the values 1 to 3 . In this paper, the positive $x_{3}$-axis is pointing downward. The temporal Fourier transform is defined as

$$
p(\mathbf{x}, \omega)=\int_{-\infty}^{\infty} p(\mathbf{x}, t) \exp (-j \omega t) \mathrm{d} t,
$$

where $\omega$ is the angular frequency and $j$ is the imaginary unit $(j=\sqrt{-1})$. To keep the notation simple, the same symbol is used for time- and frequency-domain functions (here $p$ ). The downward and upward propagating, mutually coupled, constituents of the wave field are denoted by $p^{+}$and $p^{-}$, respectively. In the space-frequency domain, the formal relation between one-way (i.e., down-going and up-going) and two-way (i.e., total) wave fields is given by ${ }^{24-31}$

$$
\left(\begin{array}{c}
p \\
v_{3}
\end{array}\right)=\left(\begin{array}{cc}
\mathcal{L}_{1} & \mathcal{L}_{1} \\
\mathcal{L}_{2} & -\mathcal{L}_{2}
\end{array}\right)\left(\begin{array}{c}
p^{+} \\
p^{-}
\end{array}\right) .
$$

Here $\mathcal{L}_{1}$ and $\mathcal{L}_{2}$ are pseudo-differential operators, which are discussed in detail in the aforementioned references. Assuming the one-way wave fields are normalized with respect to acoustic power flux, the inverse of Eq. (2) is given by

$$
\left(\begin{array}{c}
p^{+} \\
p^{-}
\end{array}\right)=\left(\begin{array}{cc}
\mathcal{L}_{2}^{t} & \mathcal{L}_{1}^{t} \\
\mathcal{L}_{2}^{t} & -\mathcal{L}_{1}^{t}
\end{array}\right)\left(\begin{array}{c}
p \\
v_{3}
\end{array}\right),
$$

where the superscript $t$ stands for operator transposition. ${ }^{28,31}$ The symmetry between the composition and decomposition 
Eqs. (2) and (3) implies that the flux-normalized one-way wave fields, as well as the corresponding one-way Green's functions and the reflection and transmission responses, obey simple reciprocity relations. ${ }^{32-36}$ This facilitates the derivation of the 3D Marchenko equation. Therefore, in the main text of this paper (except in Sec. VIII) all one-way wave fields are flux-normalized. In the Appendix the 3D Marchenko equation is derived using pressure normalized one-way wave fields.

Consider an inhomogeneous lossless medium below an acoustically transparent boundary $\partial \mathbb{D}_{0}$. The upper half-space above $\partial \mathbb{D}_{0}$ is homogeneous. The propagation velocity and mass density of the inhomogeneous medium below $\partial \mathbb{D}_{0}$ are defined as $c(\mathbf{x})$ and $\rho(\mathbf{x})$, respectively. From here onward, the spatial coordinate vector $\mathbf{x}$ is defined as $\mathbf{x}=\left(\mathbf{x}_{\mathrm{H}}, x_{3}\right)$, in which $\mathbf{x}_{\mathrm{H}}=\left(x_{1}, x_{2}\right)$ is the horizontal coordinate vector and $x_{3}$ is the vertical coordinate.

The boundary $\partial \mathbb{D}_{0}$ is chosen at depth level $x_{3}=x_{3,0}$. Coordinates at $\partial \mathbb{D}_{0}$ are denoted as $\mathbf{x}_{0}=\left(\mathbf{x}_{\mathrm{H}}, x_{3,0}\right)$. A second boundary $\partial \mathbb{D}_{i}$ is defined at an arbitrary depth level $x_{3}=x_{3, i}$, with $x_{3, i}>x_{3,0}$. Coordinates at $\partial \mathbb{D}_{i}$ are denoted as $\mathbf{x}_{i}=\left(\mathbf{x}_{\mathrm{H}}\right.$, $\left.x_{3, i}\right)$. Consequently, the notations $p\left(\mathbf{x}_{0}\right)$ and $p\left(\mathbf{x}_{i}\right)$ stand for $\left.p(\mathbf{x})\right|_{x_{3}=x_{3,0}}$ and $\left.p(\mathbf{x})\right|_{x_{3}=x_{3, i},}$, respectively [i.e., the function $p(\mathbf{x})$ evaluated at depth levels $x_{3,0}$ and $x_{3, i}$, respectively]. The domain enclosed by boundaries $\partial \mathbb{D}_{0}$ and $\partial \mathbb{D}_{i}$ is denoted as $\mathbb{D}$.

Acoustic reciprocity theorems formulate general relations between two acoustic states in one and the same domain. ${ }^{37,38}$ Flux-normalized one-way wave fields obey in the space frequency domain the following reciprocity relation: ${ }^{36,39}$

$$
\int_{\partial \mathbb{D}_{0}}\left\{p_{A}^{+} p_{B}^{-}-p_{A}^{-} p_{B}^{+}\right\} \mathrm{d} \mathbf{x}_{0}=\int_{\partial \mathbb{D}_{i}}\left\{p_{A}^{+} p_{B}^{-}-p_{A}^{-} p_{B}^{+}\right\} \mathrm{d} \mathbf{x}_{i},
$$

where subscripts $A$ and $B$ refer to two independent states. The underlying assumptions are that there are no sources in the domain $\mathbb{D}$ between $\partial \mathbb{D}_{0}$ and $\partial \mathbb{D}_{i}$ and that the medium parameters in $\mathbb{D}$ are the same in states $A$ and $B$. Note that sources may be present outside $\mathbb{D}$ and the medium parameters in the two states may differ from each other outside $\mathbb{D}$ (this is because in the derivation of reciprocity theorems only the situation inside the considered domain matters ${ }^{36-38}$ ). According to the definitions of $\mathbf{x}_{0}$ and $\mathbf{x}_{i}$, the integrations in Eq. (4) take place at $\partial \mathbb{D}_{0}$ and $\partial \mathbb{D}_{i}$, respectively, along the horizontal coordinate vector $\mathbf{x}_{\mathrm{H}}$. Equation (4) is the so-called one-way reciprocity theorem of the convolution type, because products like $p_{A}^{+} p_{B}^{-}$in the frequency domain correspond to convolutions in the time domain. A second reciprocity theorem for one-way wave fields is given by

$$
\begin{aligned}
\int_{\partial \mathbb{D}_{0}}\left\{\left(p_{A}^{+}\right)^{*} p_{B}^{+}-\left(p_{A}^{-}\right)^{*} p_{B}^{-}\right\} \mathrm{d} \mathbf{x}_{0} \\
=\int_{\partial \mathbb{D}_{i}}\left\{\left(p_{A}^{+}\right)^{*} p_{B}^{+}-\left(p_{A}^{-}\right)^{*} p_{B}^{-}\right\} \mathrm{d} \mathbf{x}_{i},
\end{aligned}
$$

where the asterisk $(*)$ denotes complex conjugation. This is the so-called one-way reciprocity theorem of the correlation type, because products like $\left(p_{A}^{+}\right)^{*} p_{B}^{+}$in the frequency domain correspond to correlations in the time domain. In addition to the assumptions mentioned above, evanescent waves are neglected in Eq. (5) at the boundaries $\partial \mathbb{D}_{0}$ and $\partial \mathbb{D}_{i}$. In Secs. IV and V, the reciprocity theorems (4) and (5) are used to derive relations between focusing functions and the Green's function of the inhomogeneous medium below $\partial \mathbb{D}_{0}$.

\section{FOCUSING FUNCTIONS}

In the derivation of the Marchenko equation for 1D scattering problems, the so-called "fundamental solutions" of the source-free Schrödinger equation play an essential role. ${ }^{1}$ For the situation of a localized scattering potential in a homogeneous embedding, the fundamental solutions $f_{1}(x, t)$ and $f_{2}(x, t)$ of the Schrödinger equation are those solutions that reduce to outgoing waves for $x \rightarrow \infty$ and $x \rightarrow-\infty$, respectively. Any other solution can be expressed as a linear combination of the two fundamental solutions.

The concept of fundamental solutions has been extended for the 3D situation. ${ }^{40}$ Here, a slightly different approach is followed. First, the 3D versions of the fundamental solutions are renamed as "focusing functions," which better reflects the character of these functions. Second, the 3D focusing functions are defined in a reference configuration rather than in the actual medium, similar as proposed by Slob et al. ${ }^{41}$ for the 1D situation. The reference configuration is chosen such that above boundary $\partial \mathbb{D}_{i}$ it is equal to the actual medium discussed in Sec. III, whereas below this boundary it is taken reflection-free. The focusing function $f_{1}(\mathbf{x}, t)$, with $\mathbf{x} \in \mathbb{R}^{3}$ and $t \in \mathbb{R}$, consists in the homogeneous upper half-space $x_{3} \leq x_{3,0}$ of a down-going field $f_{1}^{+}(\mathbf{x}, t)$ and an up-going field $f_{1}^{-}(\mathbf{x}, t)$, with $f_{1}^{+}(\mathbf{x}, t)$ shaped such that $f_{1}(\mathbf{x}, t)$ focuses at $\mathbf{x}_{i}^{\prime}$ $=\left(\mathbf{x}_{\mathrm{H}}^{\prime}, x_{3, i}\right)$ at $t=0$, and continues as a diverging down-going field $f_{1}^{+}(\mathbf{x}, t)$ into the reflection-free reference half-space $x_{3} \geq x_{3, i}$. The focusing condition is formally defined as

$$
f_{1}\left(\mathbf{x}_{\mathrm{H}}, x_{3}=x_{3, i}, t\right)=\delta\left(\mathbf{x}_{\mathrm{H}}-\mathbf{x}_{\mathrm{H}}^{\prime}\right) \delta(t),
$$

where $\delta\left(\mathbf{x}_{\mathrm{H}}-\mathbf{x}_{\mathrm{H}}^{\prime}\right)$ and $\delta(t)$ are two-dimensional (2D) and 1D Dirac delta functions, respectively. For the following derivations, it is necessary to consider the lateral position $\mathbf{x}_{\mathrm{H}}^{\prime}$ of the focal point as a variable and therefore it is useful to include the focal point $\mathbf{x}_{i}^{\prime}$ in the argument list of the wave fields. Hence, $f_{1}^{ \pm}(\mathbf{x}, t)$ is from here onward denoted as $f_{1}^{ \pm}\left(\mathbf{x}, \mathbf{x}_{i}^{\prime}, t\right)$, etc., see Fig. 3(a).

The second focusing function $f_{2}(\mathbf{x}, t)$ consists in the reflection-free reference half-space $x_{3} \geq x_{3, i}$ of an up-going field $f_{2}^{-}(\mathbf{x}, t)$ and a down-going field $f_{2}^{+}(\mathbf{x}, t)$, with $f_{2}^{-}(\mathbf{x}, t)$ shaped such that $f_{2}(\mathbf{x}, t)$ focuses at $\mathbf{x}_{0}^{\prime \prime}=\left(\mathbf{x}_{\mathrm{H}}^{\prime \prime}, x_{3,0}\right)$ at $t=0$, and continues as a diverging up-going field $f_{2}^{-}(\mathbf{x}, t)$ into the homogeneous upper half-space $x_{3} \leq x_{3,0}$. The focusing condition is formally defined as

$$
f_{2}\left(\mathbf{x}_{\mathrm{H}}, x_{3}=x_{3,0}, t\right)=\delta\left(\mathbf{x}_{\mathrm{H}}-\mathbf{x}_{\mathrm{H}}^{\prime \prime}\right) \delta(t) .
$$

From here onward the focal point $\mathbf{x}_{0}^{\prime \prime}$ is included in the argument list, hence $f_{2}^{ \pm}(\mathbf{x}, t)$ is denoted as $f_{2}^{ \pm}\left(\mathbf{x}, \mathbf{x}_{0}^{\prime \prime}, t\right)$, etc., see Fig. 3(b). 
(a)

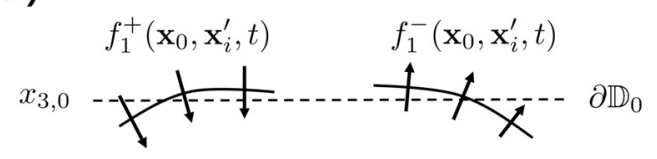

Actual inhomogeneous medium

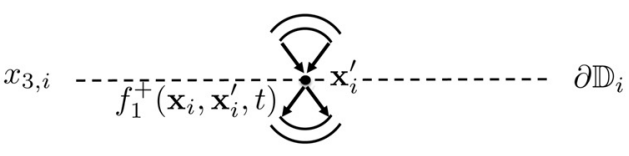

Reflection-free reference half-space (b) Homogeneous half-space

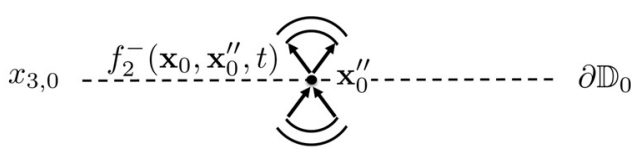

Actual inhomogeneous medium

$\mathbb{D}$

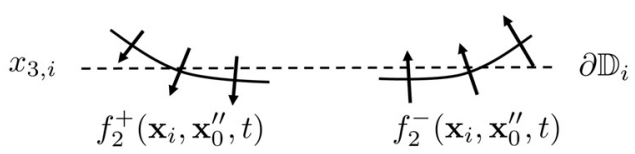

Reflection-free reference half-space

FIG. 3. 3D focusing functions in a reference configuration, which is equal to the actual medium above $\partial \mathbb{D}_{i}$ and reflection-free below $\partial \mathbb{D}_{i}$. (a) The function $f_{1}$ focuses at $\mathbf{x}_{i}^{\prime}=\left(\mathbf{x}_{\mathrm{H}}^{\prime}, x_{3, i}\right)$ at $t=0$ and continues as a diverging down-going field $f_{1}^{+}$into the lower half-space. (b) The function $f_{2}$ focuses at $\mathbf{x}_{0}^{\prime \prime}=\left(\mathbf{x}_{\mathrm{H}}^{\prime \prime}, x_{3,0}\right)$ at $t=0$ and continues as a diverging up-going field $f_{2}^{-}$into the upper half-space.

In the frequency domain, the first focusing function is written as

$$
f_{1}\left(\mathbf{x}, \mathbf{x}_{i}^{\prime}, \omega\right)=f_{1}^{+}\left(\mathbf{x}, \mathbf{x}_{i}^{\prime}, \omega\right)+f_{1}^{-}\left(\mathbf{x}, \mathbf{x}_{i}^{\prime}, \omega\right)
$$

with $f_{1}^{-}\left(\mathbf{x}, \mathbf{x}_{i}^{\prime}, \omega\right)=0$ for $x_{3} \geq x_{3, i}$. Focusing condition (6) reads in the frequency domain

$$
f_{1}\left(\mathbf{x}_{i}, \mathbf{x}_{i}^{\prime}, \omega\right)=f_{1}^{+}\left(\mathbf{x}_{i}, \mathbf{x}_{i}^{\prime}, \omega\right)=\delta\left(\mathbf{x}_{\mathrm{H}}-\mathbf{x}_{\mathrm{H}}^{\prime}\right)
$$

Note that $f_{1}\left(\mathbf{x}, \mathbf{x}_{i}^{\prime}, \omega\right)$ can only collapse into a delta function at $x_{3}=x_{3, i}$ when the entire evanescent field is included. Since the evanescent field decays exponentially during downward propagation and should remain finite at $x_{3}=x_{3, i}$, the illuminating field $f_{1}^{+}\left(\mathbf{x}, \mathbf{x}_{i}^{\prime}, \omega\right)$ in the upper-half-space $x_{3} \leq x_{3,0}$ should have very high amplitudes, which could make this focusing function unstable. To avoid this unstable behavior, evanescent waves are excluded at the focusing depth level $x_{3}=x_{3, i}$ (i.e., at boundary $\partial \mathbb{D}_{i}$ ). This means that the delta function in Eq. (9) and in subsequent equations should be interpreted as a spatially band limited delta function. Note that the sifting property of the delta function, $h\left(\mathbf{x}_{\mathrm{H}}\right)=\int \delta\left(\mathbf{x}_{\mathrm{H}}-\mathbf{x}_{\mathrm{H}}^{\prime}\right) h\left(\mathbf{x}_{\mathrm{H}}^{\prime}\right) \mathrm{d} \mathbf{x}_{\mathrm{H}}^{\prime}$, remains valid for a spatially band limited delta function, assuming $h\left(\mathbf{x}_{\mathrm{H}}\right)$ is also spatially band limited (which is the case when evanescent waves are excluded).

The second focusing function reads in the frequency domain

$$
f_{2}\left(\mathbf{x}, \mathbf{x}_{0}^{\prime \prime}, \omega\right)=f_{2}^{+}\left(\mathbf{x}, \mathbf{x}_{0}^{\prime \prime}, \omega\right)+f_{2}^{-}\left(\mathbf{x}, \mathbf{x}_{0}^{\prime \prime}, \omega\right),
$$

with $f_{2}^{+}\left(\mathbf{x}, \mathbf{x}_{0}^{\prime \prime}, \omega\right)=0$ for $x_{3} \leq x_{3,0}$. Focusing condition (7) reads in the frequency domain

$$
f_{2}\left(\mathbf{x}_{0}, \mathbf{x}_{0}^{\prime \prime}, \omega\right)=f_{2}^{-}\left(\mathbf{x}_{0}, \mathbf{x}_{0}^{\prime \prime}, \omega\right)=\delta\left(\mathbf{x}_{\mathrm{H}}-\mathbf{x}_{\mathrm{H}}^{\prime \prime}\right) .
$$

Evanescent waves are excluded at the focusing depth level $x_{3}=x_{3,0}$ (i.e., at boundary $\partial \mathbb{D}_{0}$ ); hence, the delta function should again be interpreted as a spatially band limited delta function.

Reciprocity theorems (4) and (5) can be used to find relations between the focusing functions at the boundaries $\partial \mathbb{D}_{0}$ and $\partial \mathbb{D}_{i}$. Substituting $p_{A}^{ \pm}(\mathbf{x}, \omega)=f_{1}^{ \pm}\left(\mathbf{x}, \mathbf{x}_{i}^{\prime}, \omega\right)$ and $p_{B}^{ \pm}(\mathbf{x}, \omega)=f_{2}^{ \pm}\left(\mathbf{x}, \mathbf{x}_{0}^{\prime \prime}, \omega\right)$ into Eqs. (4) and (5), using Eqs. (9) and (11), yields

$$
f_{1}^{+}\left(\mathbf{x}_{0}^{\prime \prime}, \mathbf{x}_{i}^{\prime}, \omega\right)=f_{2}^{-}\left(\mathbf{x}_{i}^{\prime}, \mathbf{x}_{0}^{\prime \prime}, \omega\right)
$$

and

$$
-\left\{f_{1}^{-}\left(\mathbf{x}_{0}^{\prime \prime}, \mathbf{x}_{i}^{\prime}, \omega\right)\right\}^{*}=f_{2}^{+}\left(\mathbf{x}_{i}^{\prime}, \mathbf{x}_{0}^{\prime \prime}, \omega\right)
$$

respectively.

\section{GREEN'S FUNCTION REPRESENTATIONS}

The one-way reciprocity theorems of Sec. III and the focusing functions introduced in Sec. IV are used to derive Green's function representations. To this end, the fluxnormalized one-way Green's function of the actual inhomogeneous medium is introduced, with its flux-normalized source at $\mathbf{x}_{0}^{\prime \prime}$, just above $\partial \mathbb{D}_{0}$, see Fig. 4 . This source radiates up-going waves into the homogeneous upper half-space and

Homogeneous half-space

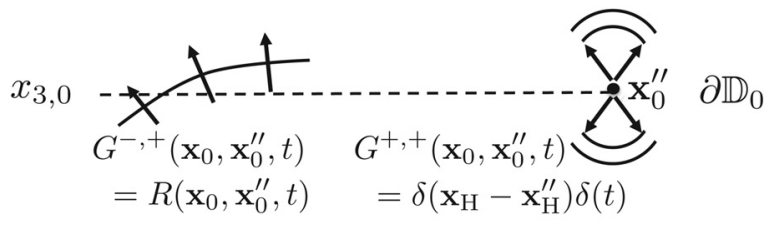

Actual inhomogeneous medium

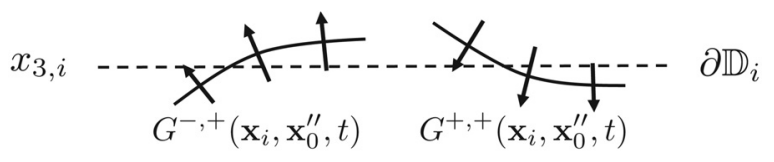

Actual inhomogeneous half-space

FIG. 4. Flux-normalized one-way Green's functions of the 3D wave equation in the actual inhomogeneous medium. The source at $\mathbf{x}_{0}^{\prime \prime}$ is chosen just above $\partial \mathbb{D}_{0}$. The Green's function $G^{-,+}\left(\mathbf{x}_{0}, \mathbf{x}_{0}^{\prime \prime}, t\right)$ is by definition the reflection response $R\left(\mathbf{x}_{0}, \mathbf{x}_{0}^{\prime \prime}, t\right)$ of the medium. 
down-going waves into the inhomogeneous lower halfspace. At and below $\partial \mathbb{D}_{0}$, the response is entirely due to the downward radiating part of the source. Directly below the source, at $\partial \mathbb{D}_{0}$, the downward propagating constituent of the Green's function is defined as

$$
G^{+,+}\left(\mathbf{x}_{0}, \mathbf{x}_{0}^{\prime \prime}, t\right)=\delta\left(\mathbf{x}_{\mathrm{H}}-\mathbf{x}_{\mathrm{H}}^{\prime \prime}\right) \delta(t) .
$$

The first superscript $(+)$ denotes the downward propagation direction at the observation point $\mathbf{x}_{0}$, whereas the second superscript $(+)$ refers to the downward radiating part of the source at $\mathbf{x}_{0}^{\prime \prime}$. Because of the flux-normalization, this direct field contribution directly below the source is simply represented by the delta function on the right-hand side. ${ }^{36}$ The upward propagating constituent of the Green's function at $\partial \mathbb{D}_{0}$ is defined as

$$
G^{-,+}\left(\mathbf{x}_{0}, \mathbf{x}_{0}^{\prime \prime}, t\right)=R\left(\mathbf{x}_{0}, \mathbf{x}_{0}^{\prime \prime}, t\right) .
$$

Here the first superscript $(-)$ denotes the upward propagation direction at the observation point $\mathbf{x}_{0}$. This Green's function is by definition the reflection response of the inhomogeneous medium, represented by $R\left(\mathbf{x}_{0}, \mathbf{x}_{0}^{\prime \prime}, t\right)$ on the right-hand side. Figure 4 further shows the downward and upward propagating constituents $G^{+,+}\left(\mathbf{x}_{i}, \mathbf{x}_{0}^{\prime \prime}, t\right)$ and $G^{-,+}\left(\mathbf{x}_{i}\right.$, $\left.\mathbf{x}_{0}^{\prime \prime}, t\right)$, respectively, at $\partial \mathbb{D}_{i}$.

In the frequency domain, the one-way Green's functions in the half-space $x_{3} \geq x_{3,0}$ are represented by $G^{ \pm,+}\left(\mathbf{x}, \mathbf{x}_{0}^{\prime \prime}, \omega\right)$, and Eqs. (14) and (15) become

$$
G^{+,+}\left(\mathbf{x}_{0}, \mathbf{x}_{0}^{\prime \prime}, \omega\right)=\delta\left(\mathbf{x}_{\mathrm{H}}-\mathbf{x}_{\mathrm{H}}^{\prime \prime}\right)
$$

and

$$
G^{-,+}\left(\mathbf{x}_{0}, \mathbf{x}_{0}^{\prime \prime}, \omega\right)=R\left(\mathbf{x}_{0}, \mathbf{x}_{0}^{\prime \prime}, \omega\right),
$$

respectively. Relations between the Green's functions $G^{ \pm,+}$ and the focusing functions $f_{1}^{ \pm}$can be found by applying the reciprocity theorems (4) and (5) to the domain $\mathbb{D}$ enclosed by $\partial \mathbb{D}_{0}$ and $\partial \mathbb{D}_{i}$. Note that this is allowed because in $\mathbb{D}$ the Green's function and the focusing function are both defined in the actual medium; the fact that below $\partial \mathbb{D}_{i}$ (i.e., outside D) the media are different does not matter. Substituting $p_{A}^{ \pm}(\mathbf{x}, \omega)=f_{1}^{ \pm}\left(\mathbf{x}, \mathbf{x}_{i}^{\prime}, \omega\right)$ and $p_{B}^{ \pm}(\mathbf{x}, \omega)=G^{ \pm,+}\left(\mathbf{x}, \mathbf{x}_{0}^{\prime \prime}, \omega\right)$ into Eqs. (4) and (5), using $R\left(\mathbf{x}_{0}, \mathbf{x}_{0}^{\prime \prime}, \omega\right)=R\left(\mathbf{x}_{0}^{\prime \prime}, \mathbf{x}_{0}, \omega\right)$ (Ref. 39) and Eqs. (9), (16), and (17), yields

$$
\begin{aligned}
& \int_{\partial \mathbb{D}_{0}} f_{1}^{+}\left(\mathbf{x}_{0}, \mathbf{x}_{i}^{\prime}, \omega\right) R\left(\mathbf{x}_{0}^{\prime \prime}, \mathbf{x}_{0}, \omega\right) \mathrm{d} \mathbf{x}_{0} \\
& \quad-f_{1}^{-}\left(\mathbf{x}_{0}^{\prime \prime}, \mathbf{x}_{i}^{\prime}, \omega\right)=G^{-,+}\left(\mathbf{x}_{i}^{\prime}, \mathbf{x}_{0}^{\prime \prime}, \omega\right)
\end{aligned}
$$

and

$$
\begin{aligned}
& -\int_{\partial \mathbb{D}_{0}}\left\{f_{1}^{-}\left(\mathbf{x}_{0}, \mathbf{x}_{i}^{\prime}, \omega\right)\right\}^{*} R\left(\mathbf{x}_{0}^{\prime \prime}, \mathbf{x}_{0}, \omega\right) \mathrm{d} \mathbf{x}_{0} \\
& +\left\{f_{1}^{+}\left(\mathbf{x}_{0}^{\prime \prime}, \mathbf{x}_{i}^{\prime}, \omega\right)\right\}^{*}=G^{+,+}\left(\mathbf{x}_{i}^{\prime}, \mathbf{x}_{0}^{\prime \prime}, \omega\right),
\end{aligned}
$$

respectively. Adding these expressions, using Eqs. (10), (12), and (13), and bringing the Green's functions to the lefthand side, gives

$$
\begin{aligned}
& G^{+,+}\left(\mathbf{x}_{i}^{\prime}, \mathbf{x}_{0}^{\prime \prime}, \omega\right)+G^{-,+}\left(\mathbf{x}_{i}^{\prime}, \mathbf{x}_{0}^{\prime \prime}, \omega\right) \\
& \quad=\int_{\partial \mathbb{D}_{0}} f_{2}\left(\mathbf{x}_{i}^{\prime}, \mathbf{x}_{0}, \omega\right) R\left(\mathbf{x}_{0}^{\prime \prime}, \mathbf{x}_{0}, \omega\right) \mathrm{d} \mathbf{x}_{0}+\left\{f_{2}\left(\mathbf{x}_{i}^{\prime}, \mathbf{x}_{0}^{\prime \prime}, \omega\right)\right\}^{*}
\end{aligned}
$$

Note that the left-hand side represents a superposition of the flux-normalized down-going and up-going Green's wave fields at $\mathbf{x}_{i}^{\prime}$, related to the downward radiating part of the source at $\mathbf{x}_{0}^{\prime \prime}$. It can be transformed into the pressurenormalized two-way Green's function $G\left(\mathbf{x}_{i}^{\prime}, \mathbf{x}_{0}^{\prime \prime}, \omega\right)$ as follows. Applying the operator $\mathcal{L}_{1}$ at depth level $x_{3}=x_{3, i}$ to the left-hand side of Eq. (20) gives, analogous to Eq. (2), the two-way Green's pressure at $\mathbf{x}_{i}^{\prime}$. This operation does not affect the source at $\mathbf{x}_{0}^{\prime \prime}$, hence, the result is written as $G^{p,+}\left(\mathbf{x}_{i}^{\prime}, \mathbf{x}_{0}^{\prime \prime}, \omega\right)$ (superscript $p$ refers to the pressure at the observation point $\mathbf{x}_{i}^{\prime}$ ). Using a combination of two-way and one-way source-receiver reciprocity, ${ }^{36,38}$ this is equal to $G^{-, q}\left(\mathbf{x}_{0}^{\prime \prime}, \mathbf{x}_{i}^{\prime}, \omega\right)$, i.e., the flux-normalized upward propagating Green's wave field at $\mathbf{x}_{0}^{\prime \prime}$, due to a source of the volume-injection type (denoted by superscript $q$ ) at $\mathbf{x}_{i}^{\prime}$. Applying the operator $\mathcal{L}_{1}$ at depth level $x_{3}=x_{3,0}$ turns this into $G^{p, q}\left(\mathbf{x}_{0}^{\prime \prime}, \mathbf{x}_{i}^{\prime}, \omega\right)$, i.e., the pressure-normalized two-way Green's function. Combining these steps, applying source-receiver reciprocity once more and dropping the superscripts $p$ and $q$ gives

$$
\begin{aligned}
G\left(\mathbf{x}_{i}^{\prime}, \mathbf{x}_{0}^{\prime \prime}, \omega\right)= & G\left(\mathbf{x}_{0}^{\prime \prime}, \mathbf{x}_{i}^{\prime}, \omega\right) \\
= & \mathcal{L}_{1}\left(x_{3, i}\right) \mathcal{L}_{1}\left(x_{3,0}\right)\left\{G^{+,+}\left(\mathbf{x}_{i}^{\prime}, \mathbf{x}_{0}^{\prime \prime}, \omega\right)\right. \\
& \left.+G^{-,+}\left(\mathbf{x}_{i}^{\prime}, \mathbf{x}_{0}^{\prime \prime}, \omega\right)\right\}
\end{aligned}
$$

Summarizing, Eqs. (20) and (21) show that the Green's function of the actual inhomogeneous medium, propagating from a source just above the surface $\partial \mathbb{D}_{0}$ (Fig. 4) to a receiver at an arbitrary depth level $\partial \mathbb{D}_{i}$ (or vice versa), can be obtained from the reflection response of the actual medium, observed at the surface $\partial \mathbb{D}_{0}$, and the focusing function $f_{2}$ of the reference configuration [Fig. 3(b)].

\section{3D MARCHENKO EQUATION}

The right-hand side of Eq. (20) is used to derive the 3D Marchenko equation, which is an integral equation for the focusing function $f_{2}$. Transforming Eq. (20) to the time domain gives

$$
\begin{aligned}
G^{+,+} & \left(\mathbf{x}_{i}^{\prime}, \mathbf{x}_{0}^{\prime \prime}, t\right)+G^{-,+}\left(\mathbf{x}_{i}^{\prime}, \mathbf{x}_{0}^{\prime \prime}, t\right) \\
= & \int_{\partial \mathbb{D}_{0}} \mathrm{~d} \mathbf{x}_{0} \int_{-\infty}^{t} f_{2}\left(\mathbf{x}_{i}^{\prime}, \mathbf{x}_{0}, t^{\prime}\right) R\left(\mathbf{x}_{0}^{\prime \prime}, \mathbf{x}_{0}, t-t^{\prime}\right) \mathrm{d} t^{\prime} \\
& \quad+f_{2}\left(\mathbf{x}_{i}^{\prime}, \mathbf{x}_{0}^{\prime \prime},-t\right) .
\end{aligned}
$$

The upper integration limit $\left(t^{\prime}=t\right)$ stems from the causality of the reflection response $R\left(\mathbf{x}_{0}^{\prime \prime}, \mathbf{x}_{0}, t-t^{\prime}\right)$. Let $t_{\mathrm{d}}\left(\mathbf{x}_{i}^{\prime}, \mathbf{x}_{0}^{\prime \prime}\right)$ denote the travel time of the first arrival between $\mathbf{x}_{0}^{\prime \prime}$, located just above $\partial \mathbb{D}_{0}$, and $\mathbf{x}_{i}^{\prime}$ at $\partial \mathbb{D}_{i}$ [in case of a triplicated wave, $t_{\mathrm{d}}\left(\mathbf{x}_{i}^{\prime}, \mathbf{x}_{0}^{\prime \prime}\right)$ is the travel time of the first onset]. In the following, Eq. (22) is evaluated only for $t<t_{\mathrm{d}}\left(\mathbf{x}_{i}^{\prime}, \mathbf{x}_{0}^{\prime \prime}\right)$, hence 


$$
\begin{aligned}
0= & \int_{\partial \mathbb{D}_{0}} \mathrm{~d} \mathbf{x}_{0} \int_{-\infty}^{t} f_{2}\left(\mathbf{x}_{i}^{\prime}, \mathbf{x}_{0}, t^{\prime}\right) R\left(\mathbf{x}_{0}^{\prime \prime}, \mathbf{x}_{0}, t-t^{\prime}\right) \mathrm{d} t^{\prime} \\
& +f_{2}\left(\mathbf{x}_{i}^{\prime}, \mathbf{x}_{0}^{\prime \prime},-t\right), \text { for } t<t_{\mathrm{d}}\left(\mathbf{x}_{i}^{\prime}, \mathbf{x}_{0}^{\prime \prime}\right) .
\end{aligned}
$$

Assuming $R\left(\mathbf{x}_{0}^{\prime \prime}, \mathbf{x}_{0}, t\right)$ is known (obtained from reflection measurements at the surface), the aim is to determine the focusing function $f_{2}\left(\mathbf{x}_{i}, \mathbf{x}_{0}^{\prime \prime}, t\right)$. Equation (23) has no unique solution (e.g., $f_{2}=0$ is also a solution). Therefore an ansatz will be made for the form of $f_{2}\left(\mathbf{x}_{i}, \mathbf{x}_{0}^{\prime \prime}, t\right)$, inspired by the 1D case. In the $1 \mathrm{D}$ derivation, ${ }^{1} f_{2}(x, t)$ is defined as a delta pulse traveling in the negative $x$-direction followed by a coda caused by the scattering potential. The coda vanishes beyond the scattering region, leaving only the delta pulse traveling in the negative $x$-direction beyond this region.

Before generalizing the ansatz to the 3D situation, an auxiliary property of $f_{2}^{-}\left(\mathbf{x}_{i}, \mathbf{x}_{0}^{\prime \prime}, t\right)$ is derived. Figure 5(a) shows the transmission response $T\left(\mathbf{x}_{i}, \mathbf{x}_{0}^{\prime \prime}, t\right)$ in the reference configuration to a source at $\mathbf{x}_{0}^{\prime \prime}$ just above $\partial \mathbb{D}_{0}$. Hence, $T\left(\mathbf{x}_{i}\right.$, $\left.\mathbf{x}_{0}^{\prime \prime}, t\right)$ is the transmission of the actual inhomogeneous medium in $\mathbb{D}$, sandwiched between a homogeneous half-space above $\partial \mathbb{D}_{0}$ and a reflection-free reference half-space below $\partial \mathbb{D}_{i}$. Substituting $p_{A}^{+}\left(\mathbf{x}_{0}, \omega\right)=\delta\left(\mathbf{x}_{H}-\mathbf{x}_{\mathrm{H}}^{\prime \prime}\right), p_{A}^{+}\left(\mathbf{x}_{i}, \omega\right)=T\left(\mathbf{x}_{i}\right.$, $\left.\mathbf{x}_{0}^{\prime \prime}, \omega\right), p_{A}^{-}\left(\mathbf{x}_{i}, \omega\right)=0$, and $p_{B}^{ \pm}(\mathbf{x}, \omega)=f_{2}^{ \pm}\left(\mathbf{x}, \mathbf{x}_{0}^{\prime}, \omega\right)$ into Eq. (4), using Eq. (11) (with single instead of double primes), yields

$$
\delta\left(\mathbf{x}_{\mathrm{H}}^{\prime}-\mathbf{x}_{\mathrm{H}}^{\prime \prime}\right)=\int_{\partial \mathbb{D}_{i}} T\left(\mathbf{x}_{i}, \mathbf{x}_{0}^{\prime \prime}, \omega\right) f_{2}^{-}\left(\mathbf{x}_{i}, \mathbf{x}_{0}^{\prime}, \omega\right) \mathrm{d} \mathbf{x}_{i} .
$$

Hence, $f_{2}^{-}\left(\mathbf{x}_{i}, \mathbf{x}_{0}^{\prime \prime}, \omega\right)$ is the inverse of the transmission response $T\left(\mathbf{x}_{i}, \mathbf{x}_{0}^{\prime \prime}, \omega\right)$ in the sense of Eq. (24). In the time domain, this is formulated as

$$
f_{2}^{-}\left(\mathbf{x}_{i}, \mathbf{x}_{0}^{\prime \prime}, t\right)=T^{\text {inv }}\left(\mathbf{x}_{i}, \mathbf{x}_{0}^{\prime \prime}, t\right) .
$$

Analogous to the $1 \mathrm{D}$ case, the ansatz for the 3D situation is that $f_{2}\left(\mathbf{x}_{i}, \mathbf{x}_{0}^{\prime \prime}, t\right)$ can be defined as the superposition of a direct up-going wave and a scattering coda, which vanishes in the homogeneous upper half-space. Since $f_{2}\left(\mathbf{x}_{i}, \mathbf{x}_{0}^{\prime \prime}, t\right)$ focuses at $\mathbf{x}_{0}^{\prime \prime}$ at $t=0$ [see Eq. (7) and Fig. 3(b)], it is reasonable to assume that the first event arriving at $\mathbf{x}_{0}^{\prime \prime}$ is the direct arrival of the up-going field $f_{2}^{-}\left(\mathbf{x}_{i}, \mathbf{x}_{0}^{\prime \prime}, t\right)$, i.e., the direct arrival of the inverse transmission response, which is denoted as $T_{\mathrm{d}}^{\text {inv }}\left(\mathbf{x}_{i}\right.$, $\left.\mathbf{x}_{0}^{\prime \prime}, t\right)$. The travel time of this direct arrival is $-t_{\mathrm{d}}\left(\mathbf{x}_{i}, \mathbf{x}_{0}^{\prime \prime}\right)$. Figure 5(b) illustrates the propagation of $T_{\mathrm{d}}^{\text {inv }}\left(\mathbf{x}, \mathbf{x}_{0}^{\prime \prime}, t\right)$ through the inhomogeneous medium and its focusing at $\mathbf{x}_{0}^{\prime \prime}$ at $t=0$. The ansatz for $f_{2}\left(\mathbf{x}_{i}, \mathbf{x}_{0}^{\prime \prime}, t\right)$ is thus written as

$$
f_{2}\left(\mathbf{x}_{i}, \mathbf{x}_{0}^{\prime \prime}, t\right)=T_{\mathrm{d}}^{\text {inv }}\left(\mathbf{x}_{i}, \mathbf{x}_{0}^{\prime \prime}, t\right)+M\left(\mathbf{x}_{i}, \mathbf{x}_{0}^{\prime \prime}, t\right),
$$

where $M\left(\mathbf{x}_{i}, \mathbf{x}_{0}^{\prime \prime}, t\right)$ is the coda following the direct arrival, with

$$
M\left(\mathbf{x}_{i}, \mathbf{x}_{0}^{\prime \prime}, t\right)=0, \quad \text { for } \quad t \leq-t_{\mathrm{d}}\left(\mathbf{x}_{i}, \mathbf{x}_{0}^{\prime \prime}\right) .
$$

This coda is the result of reflections taking place in the inhomogeneous medium in $\mathbb{D}$; it vanishes in the homogeneous upper half-space. Figure 5(c) illustrates the scattering coda $M\left(\mathbf{x}, \mathbf{x}_{0}^{\prime \prime}, t\right)$ related to a single ray of the direct wave. (a) Homogeneous half-space

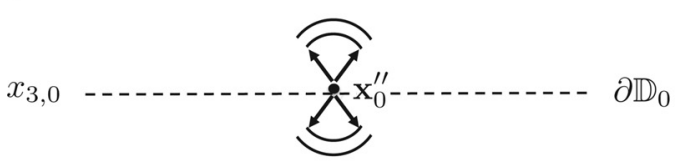

Actual inhomogeneous medium

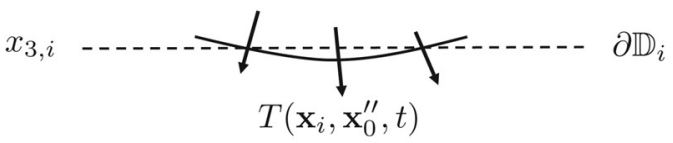

Reflection-free reference half-space

(b)

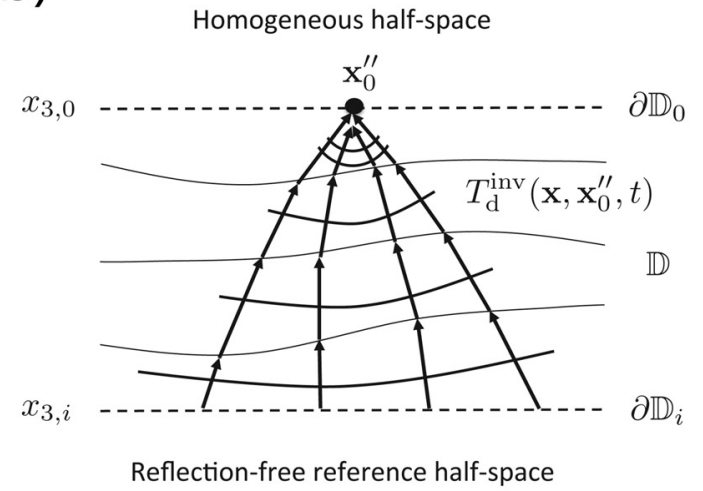

(c)

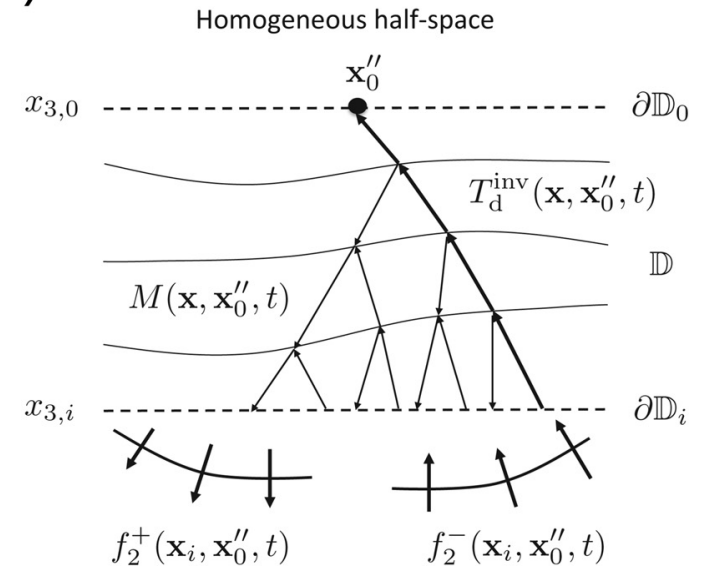

FIG. 5. (a) Transmission response $T\left(\mathbf{x}_{i}, \mathbf{x}_{0}^{\prime \prime}, t\right)$ in the reference configuration. Its inverse is equal to the up-going wave $f_{2}^{-}\left(\mathbf{x}_{i}, \mathbf{x}_{0}^{\prime \prime}, t\right)$. (b) The ansatz for the focusing function $f_{2}\left(\mathbf{x}_{i}, \mathbf{x}_{0}^{\prime \prime}, t\right)$ is that it can be defined as the superposition of a direct up-going wave and a scattering coda. The direct up-going wave consists of the direct arrival of the inverse transmission response, i.e., $T_{\mathrm{d}}^{\text {inv }}(\mathbf{x}$, $\left.\mathbf{x}_{0}^{\prime \prime}, t\right)$. (c) The coda $M\left(\mathbf{x}, \mathbf{x}_{0}^{\prime \prime}, t\right)$ is a result of scattering in the inhomogeneous medium in $\mathbb{D}$. For illustration, the fat solid ray represents the right-most ray of the direct up-going wave of (b), whereas the thin solid rays constitute the scattering coda. Note that the coda vanishes in the upper half-space.

Note that the ansatz [Eq. (26)] limits the validity of the following derivation to configurations for which the ansatz holds true. For example, it holds in layered media with moderately curved interfaces as long as $\left|\mathbf{x}_{\mathrm{H}}-\mathbf{x}_{\mathrm{H}}^{\prime \prime}\right|$ is not too large (to avoid occurrence of turning waves, head waves, etc.). The conditions underlying the ansatz need further investigation, which is beyond the scope of this paper. 
Substituting Eq. (26) into Eq. (23) yields

$$
\begin{aligned}
& \int_{\partial \mathbb{D}_{0}} \mathrm{~d} \mathbf{x}_{0} \int_{-\infty}^{-t_{\mathrm{d}}^{\epsilon}\left(\mathbf{x}_{i}^{\prime}, \mathbf{x}_{0}\right)} T_{\mathrm{d}}^{\mathrm{inv}}\left(\mathbf{x}_{i}^{\prime}, \mathbf{x}_{0}, t^{\prime}\right) R\left(\mathbf{x}_{0}^{\prime \prime}, \mathbf{x}_{0}, t-t^{\prime}\right) \mathrm{d} t^{\prime} \\
& \quad+\int_{\partial \mathbb{D}_{0}} \mathrm{~d} \mathbf{x}_{0} \int_{-t_{\mathrm{d}}^{\epsilon}\left(\mathbf{x}_{i}^{\prime}, \mathbf{x}_{0}\right)}^{t} M\left(\mathbf{x}_{i}^{\prime}, \mathbf{x}_{0}, t^{\prime}\right) R\left(\mathbf{x}_{0}^{\prime \prime}, \mathbf{x}_{0}, t-t^{\prime}\right) \mathrm{d} t^{\prime} \\
& \quad+M\left(\mathbf{x}_{i}^{\prime}, \mathbf{x}_{0}^{\prime \prime},-t\right)=0 \text { for } t<t_{\mathrm{d}}\left(\mathbf{x}_{i}^{\prime}, \mathbf{x}_{0}^{\prime \prime}\right)
\end{aligned}
$$

where $t_{\mathrm{d}}^{\epsilon}\left(\mathbf{x}_{i}^{\prime}, \mathbf{x}_{0}\right)=t_{\mathrm{d}}\left(\mathbf{x}_{i}^{\prime}, \mathbf{x}_{0}\right)-\epsilon$, with $\epsilon$ a small positive constant [it is introduced to assure that the direct arrival at $t^{\prime}=-t_{\mathrm{d}}\left(\mathbf{x}_{i}^{\prime}, \mathbf{x}_{0}\right)$ is included in the first integral]. Equation (28) is the 3D Marchenko equation. It is an integral equation for the coda $M\left(\mathbf{x}_{i}^{\prime}, \mathbf{x}_{0}^{\prime \prime}, t\right)$ of the focusing function $f_{2}\left(\mathbf{x}_{i}^{\prime}, \mathbf{x}_{0}^{\prime \prime}, t\right)$.

\section{ITERATIVE SOLUTION METHOD}

To solve the Marchenko equation for $M\left(\mathbf{x}_{i}^{\prime}, \mathbf{x}_{0}^{\prime \prime}, t\right)$, it is rewritten into the following iterative scheme:

$$
\begin{aligned}
M_{k}\left(\mathbf{x}_{i}^{\prime}, \mathbf{x}_{0}^{\prime \prime},-t\right)= & M_{0}\left(\mathbf{x}_{i}^{\prime}, \mathbf{x}_{0}^{\prime \prime},-t\right) \\
& -\int_{\partial \mathbb{D}_{0}} \mathrm{~d} \mathbf{x}_{0} \int_{-t_{\mathrm{d}}^{\epsilon}\left(\mathbf{x}_{i}^{\prime}, \mathbf{x}_{0}\right)}^{t} M_{k-1}\left(\mathbf{x}_{i}^{\prime}, \mathbf{x}_{0}, t^{\prime}\right) \\
& \times R\left(\mathbf{x}_{0}^{\prime \prime}, \mathbf{x}_{0}, t-t^{\prime}\right) \mathrm{d} t^{\prime},
\end{aligned}
$$

with

$$
\begin{aligned}
& M_{0}\left(\mathbf{x}_{i}^{\prime}, \mathbf{x}_{0}^{\prime \prime},-t\right) \\
& \quad=-\int_{\partial \mathbb{D}_{0}} \mathrm{~d} \mathbf{x}_{0} \int_{-\infty}^{-t_{\mathrm{d}}^{\epsilon}\left(\mathbf{x}_{i}^{\prime}, \mathbf{x}_{0}\right)} T_{\mathrm{d}}^{\mathrm{inv}}\left(\mathbf{x}_{i}^{\prime}, \mathbf{x}_{0}, t^{\prime}\right) R\left(\mathbf{x}_{0}^{\prime \prime}, \mathbf{x}_{0}, t-t^{\prime}\right) \mathrm{d} t^{\prime},
\end{aligned}
$$

for $t<t_{\mathrm{d}}\left(\mathbf{x}_{i}^{\prime}, \mathbf{x}_{0}^{\prime \prime}\right)$, and

$$
M_{k}\left(\mathbf{x}_{i}^{\prime}, \mathbf{x}_{0}^{\prime \prime},-t\right)=0, \quad \text { for } \quad t \geq t_{\mathrm{d}}\left(\mathbf{x}_{i}^{\prime}, \mathbf{x}_{0}^{\prime \prime}\right) .
$$

Evaluation of Eq. (30) requires that, apart from the reflection response, information about the direct arrivals of the transmission response is available. These direct arrivals can, for example, be modeled in an estimated background model. In its simplest form, $T_{\mathrm{d}}^{\text {inv }}\left(\mathbf{x}_{i}^{\prime}, \mathbf{x}_{0}, t\right)$ is approximated by $T_{\mathrm{d}}\left(\mathbf{x}_{0}, \mathbf{x}_{i}^{\prime}\right.$, $-t$ ). This approximation properly accounts for travel times and geometrical spreading, but ignores the effect of transmission losses at the interfaces. ${ }^{42}$ Using this approximation, the evaluation of Eq. (30) requires the direct arrivals between all positions $\mathbf{x}_{0}$ at the surface $\partial \mathrm{D}_{0}$ and the selected position $\mathbf{x}_{i}^{\prime}$ at $\partial \mathbb{D}_{i}$. In the more general situation, $T_{\mathrm{d}}^{\text {inv }}\left(\mathbf{x}_{i}^{\prime}, \mathbf{x}_{0}\right.$, $t)$ is an inverse in the sense of Eq. (24). Determining $T_{\mathrm{d}}^{\text {inv }}\left(\mathbf{x}_{i}^{\prime}\right.$, $\mathbf{x}_{0}, t$ ) involves resolving an integral equation (which in practice comes to matrix inversion). This requires the direct arrivals between all positions $\mathbf{x}_{0}$ at the surface $\partial \mathbb{D}_{0}$ and all positions $\mathbf{x}_{i}^{\prime}$ at $\partial \mathbb{D}_{i}$.

Once $M_{0}\left(\mathbf{x}_{i}^{\prime}, \mathbf{x}_{0}^{\prime \prime},-t\right)$ has been evaluated, it is used in the iterative scheme of Eq. (29), which starts for $k=1$ and continues until convergence. The scheme is expected to converge as long as the reflection response does not return all emitted energy back to the surface $\partial \mathbb{D}_{0}$. Using Eq. (26), the successive iterations for the focusing function $f_{2}\left(\mathbf{x}_{i}^{\prime}, \mathbf{x}_{0}^{\prime \prime}, t\right)$ read

$$
\begin{aligned}
& f_{2,0}\left(\mathbf{x}_{i}^{\prime}, \mathbf{x}_{0}^{\prime \prime}, t\right)=T_{\mathrm{d}}^{\text {inv }}\left(\mathbf{x}_{i}^{\prime}, \mathbf{x}_{0}^{\prime \prime}, t\right), \\
& f_{2, k}\left(\mathbf{x}_{i}^{\prime}, \mathbf{x}_{0}^{\prime \prime}, t\right)=T_{\mathrm{d}}^{\text {inv }}\left(\mathbf{x}_{i}^{\prime}, \mathbf{x}_{0}^{\prime \prime}, t\right)+M_{k-1}\left(\mathbf{x}_{i}^{\prime}, \mathbf{x}_{0}^{\prime \prime}, t\right) .
\end{aligned}
$$

Note that the iterative scheme of Eqs. (29)-(31) resembles the iterative time-reversal approach of Fink and coworkers. $^{43-45}$ However, whereas in the iterative timereversal approach the measurements are time-reversed and physically re-emitted into the medium, the scheme described by Eqs. (29)-(31) is implemented as a numerical processing method. Moreover, the iterative time-reversal approach is either limited to focusing on the strongest scatterer in reflection data, ${ }^{43}$ or it is applied to measured transmission data. ${ }^{44}$ In contrast, the scheme of Eqs. (29)-(31) does neither need measured transmission data nor a physical scatterer to focus on: The reflection measurements plus an estimate of the direct arrivals of the transmission response suffice to obtain the focusing function for any focal point inside the medium. Finally, the distinction between the different time intervals in Eqs. (29)-(31), which follows from the theoretical analysis, is necessary for a correct treatment of multiple scattering in the reflection data. This distinction is absent in the iterative time-reversal approach for reflection data. ${ }^{43}$

What the time-reversal approach and the Marchenko method presented here have in common is that one does not need to know the medium. In the time-reversal approach, the waves propagate back through the physical medium, which one therefore does not need to know. In the Marchenko method presented here, one needs to know a smooth background model, but all detailed information of the medium is encoded in the measured reflected waves. The algorithm convolves these reflected waves in the appropriate way to retrieve the focusing function and, subsequently, the Green's function (see Sec. VIII).

\section{GREEN'S FUNCTION RETRIEVAL FROM REFLECTION DATA: THE MARCHENKO APPROACH}

The foregoing sections contain all the ingredients to design a scheme for iterative retrieval of the Green's function $G\left(\mathbf{x}_{0}^{\prime \prime}, \mathbf{x}_{i}^{\prime}, t\right)$, with $\mathbf{x}_{i}^{\prime}$ inside the medium, from the reflection response $R\left(\mathbf{x}_{0}^{\prime \prime}, \mathbf{x}_{0}, t\right)$ at the surface and the estimated direct arrival $T_{\mathrm{d}}\left(\mathbf{x}_{i}^{\prime}, \mathbf{x}_{0}, t\right)$. The derivation of the underlying 3D Marchenko equation is based on reciprocity theorems for flux-normalized one-way wave fields. Hence, the reflection response and direct arrival, which are required as input for the Green's function retrieval scheme, need to be flux-normalized as well. In the Appendix, a 3D Marchenko equation is derived from reciprocity theorems for pressure-normalized one-way wave fields. Green's function retrieval, based on that version of the Marchenko equation, requires a pressure-normalized reflection response and a pressure-normalized direct arrival as input. In the following, the steps needed to retrieve the Green's function from the measured reflection response at the surface and the estimated direct arrivals are presented for both approaches. 
Data-driven Green's function retrieval according to the flux-normalized approach involves the following steps.

(1) In case the reflection response is measured at a free surface, remove the surface-related multiple reflections. ${ }^{46,47}$ Deconvolve the reflection response for the source time function. The output is the reflection response in a configuration with a homogeneous half-space above the acquisition surface $\partial \mathbb{D}_{0}$.

(2) Apply flux-normalization to the reflection response and the estimated direct arrival.

(3) Evaluate the inverse direct arrival $T_{\mathrm{d}}^{\text {inv }}\left(\mathbf{x}_{i}^{\prime}, \mathbf{x}_{0}^{\prime \prime}, t\right)$. Optionally, approximate $T_{\mathrm{d}}^{\text {inv }}\left(\mathbf{x}_{i}^{\prime}, \mathbf{x}_{0}^{\prime \prime}, t\right)$ by $T_{\mathrm{d}}\left(\mathbf{x}_{0}^{\prime \prime}, \mathbf{x}_{i}^{\prime},-t\right)$.
(4) Use the scheme of Eqs. (29)-(31) to obtain the iterations $M_{k}\left(\mathbf{x}_{i}^{\prime}, \mathbf{x}_{0}^{\prime \prime}, t\right)$ for the coda of the flux-normalized focusing function. Construct the iterations $f_{2, k}\left(\mathbf{x}_{i}^{\prime}, \mathbf{x}_{0}^{\prime \prime}, t\right)$ of the flux-normalized focusing function, using Eqs. (32) and (33).

(5) After convergence, construct the flux-normalized twoway Green's function $G^{+,+}\left(\mathbf{x}_{i}^{\prime}, \mathbf{x}_{0}^{\prime \prime}, t\right)+G^{-,+}\left(\mathbf{x}_{i}^{\prime}, \mathbf{x}_{0}^{\prime \prime}, t\right)$, using Eq. (22).

(6) Construct the pressure-normalized Green's function $G\left(\mathbf{x}_{i}^{\prime}, \mathbf{x}_{0}^{\prime \prime}, t\right)=G\left(\mathbf{x}_{0}^{\prime \prime}, \mathbf{x}_{i}^{\prime}, t\right)$, using Eq. (21).

Data-driven Green's function retrieval according to the pressure-normalized approach involves the following steps. (a)

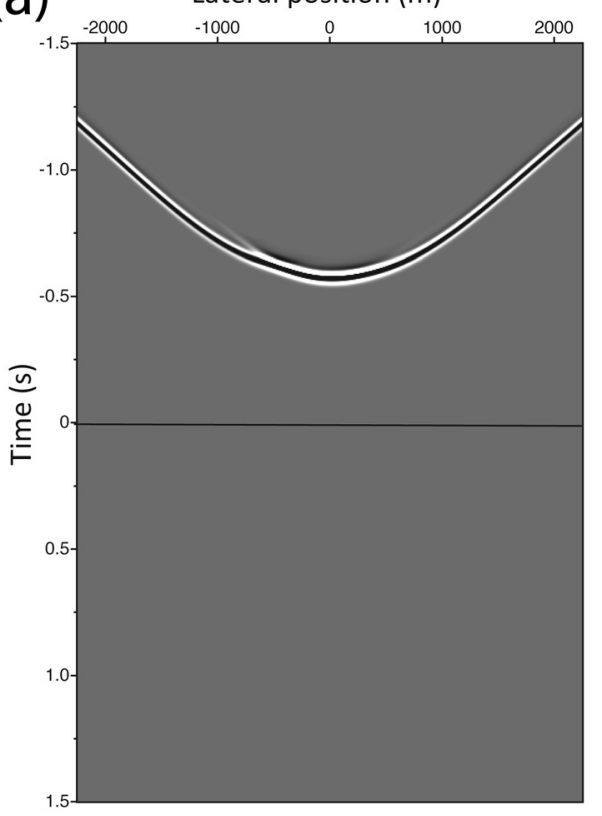

(c)

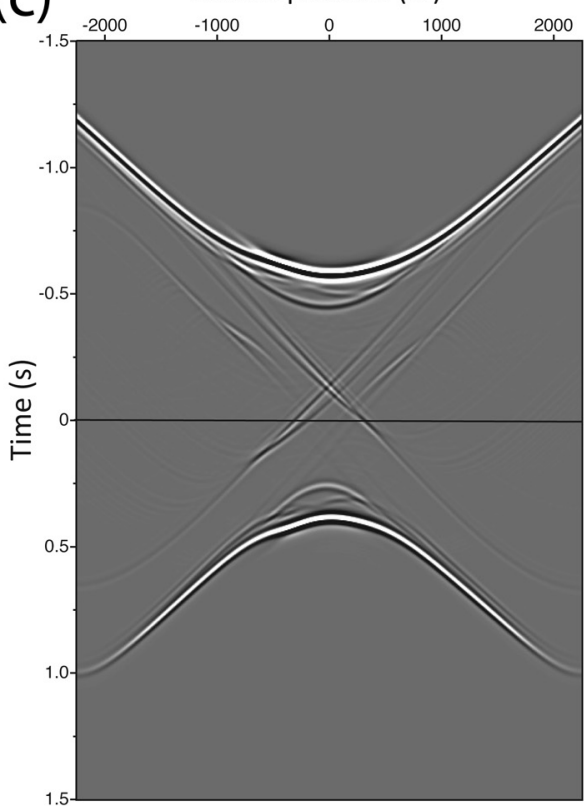

(b)

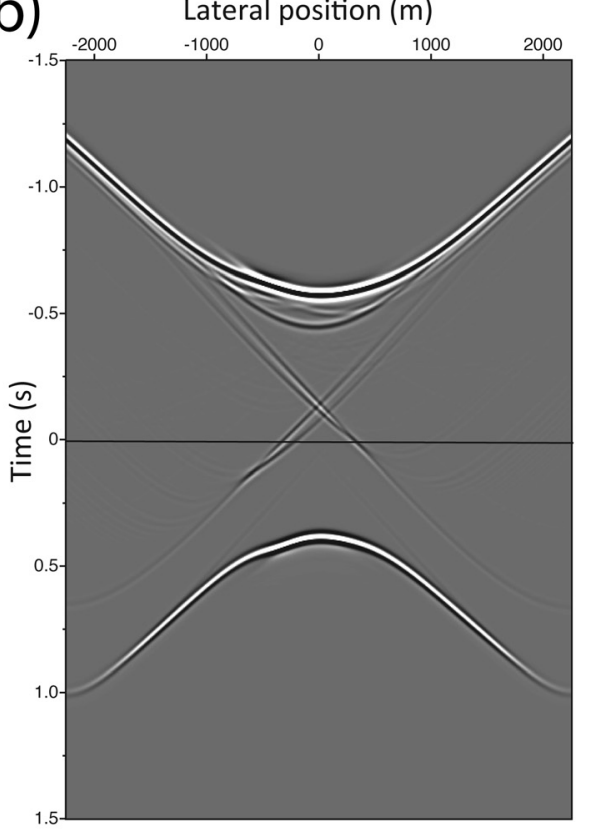

(d)

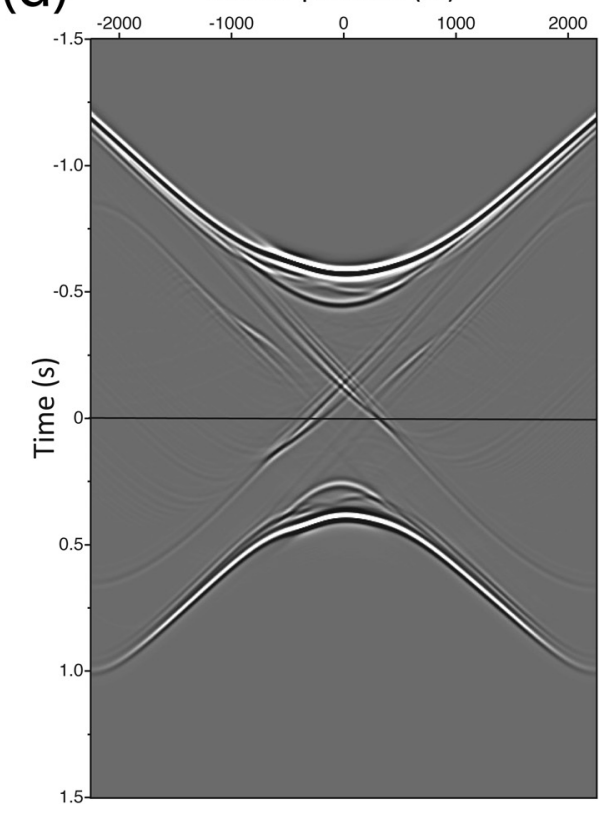

FIG. 6. The focusing function $f_{2, k}\left(\mathbf{x}_{i}^{\prime}\right.$, $\left.\mathbf{x}_{0}^{\prime \prime}, t\right)$ for fixed $\mathbf{x}_{i}^{\prime}$ and variable $\mathbf{x}_{0}^{\prime \prime}$. (a) $k=0$. (b) $k=1$. (c) $k=2$. (d) $k=10$. 
(1) As above.

(2) Evaluate the inverse direct arrival $\mathcal{T}_{\mathrm{d}}^{\text {inv }}\left(\mathbf{x}_{i}^{\prime}, \mathbf{x}_{0}^{\prime \prime}, t\right)$, where $\mathcal{T}_{\mathrm{d}}\left(\mathbf{x}_{i}^{\prime}, \mathbf{x}_{0}^{\prime \prime}, t\right)$ is related to the direct arrival of the pressure-normalized Green's function, $G_{\mathrm{d}}\left(\mathbf{x}_{i}^{\prime}, \mathbf{x}_{0}^{\prime \prime}, t\right)$, via Eqs. (A24) and (A26). Optionally, approximate $\mathcal{T}_{\mathrm{d}}^{\text {inv }}\left(\mathbf{x}_{i}^{\prime}\right.$, $\left.\mathbf{x}_{0}^{\prime \prime}, t\right)$ by $G_{\mathrm{d}}\left(\mathbf{x}_{0}^{\prime \prime}, \mathbf{x}_{i}^{\prime},-t\right)$.

(3) Use the scheme of Eqs. (A27)-(A29) to obtain the iterations $M_{k}\left(\mathbf{x}_{i}^{\prime}, \mathbf{x}_{0}^{\prime \prime}, t\right)$ for the coda of the pressure-normalized focusing function. Construct the iterations $f_{2, k}\left(\mathbf{x}_{i}^{\prime}, \mathbf{x}_{0}^{\prime \prime}, t\right)$ of the pressure-normalized focusing function, using Eqs. (A30) and (A31).

(4) After convergence, construct the pressure-normalized Green's function $G\left(\mathbf{x}_{i}^{\prime}, \mathbf{x}_{0}^{\prime \prime}, t\right)=G\left(\mathbf{x}_{0}^{\prime \prime}, \mathbf{x}_{i}^{\prime}, t\right)$, using Eqs. (A18) and (A19).

The schemes above have been derived for 3D media but hold equally well for 2D media. The latter scheme is illustrated with the 2D numerical data of Fig. 1, used earlier to illustrate a classical method for retrieving the Green's function from reflection data. Figure 1(b) represents the pressurenormalized reflection response $R\left(\mathbf{x}_{0}^{\prime \prime}, \mathbf{x}_{0}, t\right)$ for fixed $\mathbf{x}_{0}$ and variable $\mathbf{x}_{0}^{\prime \prime}$, observed at the surface $\partial \mathbb{D}_{0}$. The half-space above $\partial \mathbb{D}_{0}$ is homogeneous, hence, the reflection response contains no surface-related multiple reflections. The aim is to retrieve the Green's function $G\left(\mathbf{x}_{0}^{\prime \prime}, \mathbf{x}_{i}^{\prime}, t\right)$, with its source at $\mathbf{x}_{i}^{\prime}=(0 \mathrm{~m}, 1100 \mathrm{~m})$. Similar as for the classical method this requires, apart from the reflection response, an estimate of the direct arrival of the Green's function, $G_{\mathrm{d}}\left(\mathbf{x}_{0}^{\prime \prime}, \mathbf{x}_{i}^{\prime}, t\right)$, which is shown in Fig. 1(e) for fixed $\mathbf{x}_{i}^{\prime}$ and variable $\mathbf{x}_{0}^{\prime \prime}$. Note that a triplication is present in this estimated direct arrival, although less prominent than in the direct arrival of the Green's function in the actual medium [Fig. 1(c)]. The travel time $t_{\mathrm{d}}\left(\mathbf{x}_{0}^{\prime \prime}\right.$, $\mathbf{x}_{i}^{\prime}$ ), appearing in the iterative scheme, is defined as the time of the first onset of the triplicated event in Fig. 1(e).

Because $\partial \mathbb{D}_{0}$ is not a free surface, the surface-related multiple elimination in step 1 is skipped. For step 2 the most simple approach is chosen, i.e., $\mathcal{T}_{\mathrm{d}}^{\text {inv }}\left(\mathbf{x}_{i}^{\prime}, \mathbf{x}_{0}^{\prime \prime}, t\right)$ is approximated by $G_{\mathrm{d}}\left(\mathbf{x}_{0}^{\prime \prime}, \mathbf{x}_{i}^{\prime},-t\right)$, the time-reversal of the direct arrival of the Green's function in Fig. 1(e). This is the zeroth

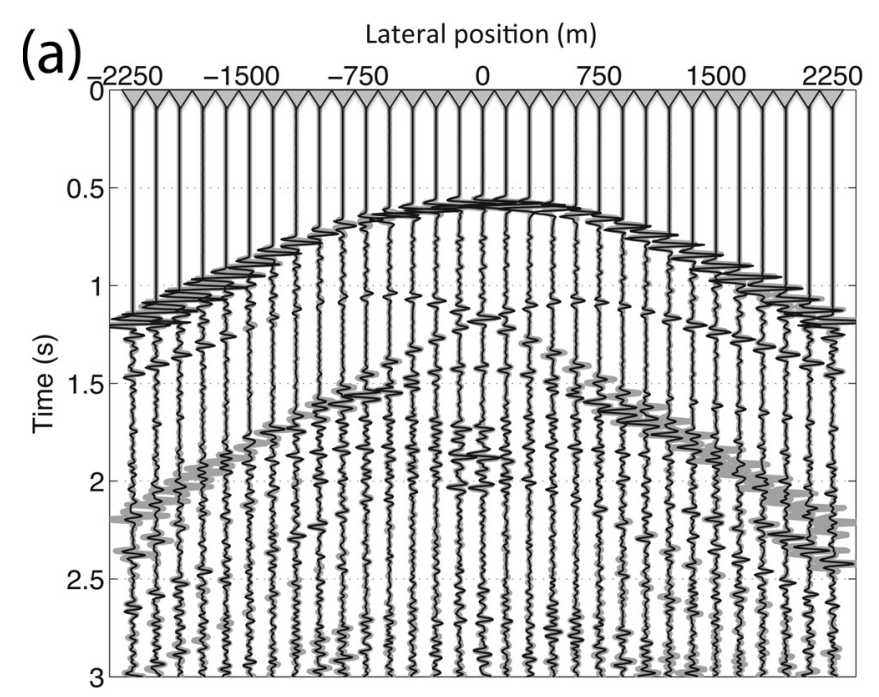

(b)

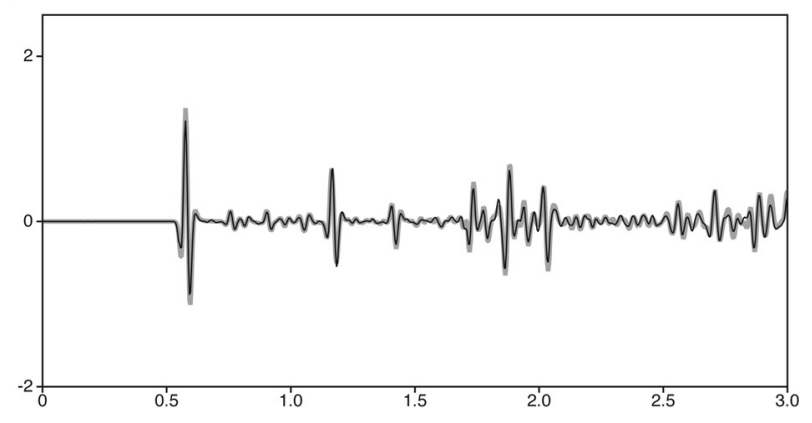

FIG. 7. Retrieved Green's function, using the Marchenko approach (thin black lines), overlain on the directly modeled Green's function (thick gray lines). (a) Green's function at all receivers in Fig. 1(a). (b) Green's function at the central receiver in Fig. 1(a).

order iteration of the pressure-normalized focusing function, $f_{2,0}\left(\mathbf{x}_{i}^{\prime}, \mathbf{x}_{0}^{\prime \prime}, t\right)$, which is shown in Fig. 6(a). Step 3 involves evaluating $f_{2, k}\left(\mathbf{x}_{i}^{\prime}, \mathbf{x}_{0}^{\prime \prime}, t\right)$ for successive values of $k$. Figures 6(b)-6(d) show $f_{2, k}\left(\mathbf{x}_{i}^{\prime}, \mathbf{x}_{0}^{\prime \prime}, t\right)$ for fixed $\mathbf{x}_{i}^{\prime}$ and variable $\mathbf{x}_{0}^{\prime \prime}$, for $k=1,2$, and 10 , respectively. Note that during the first few (a)

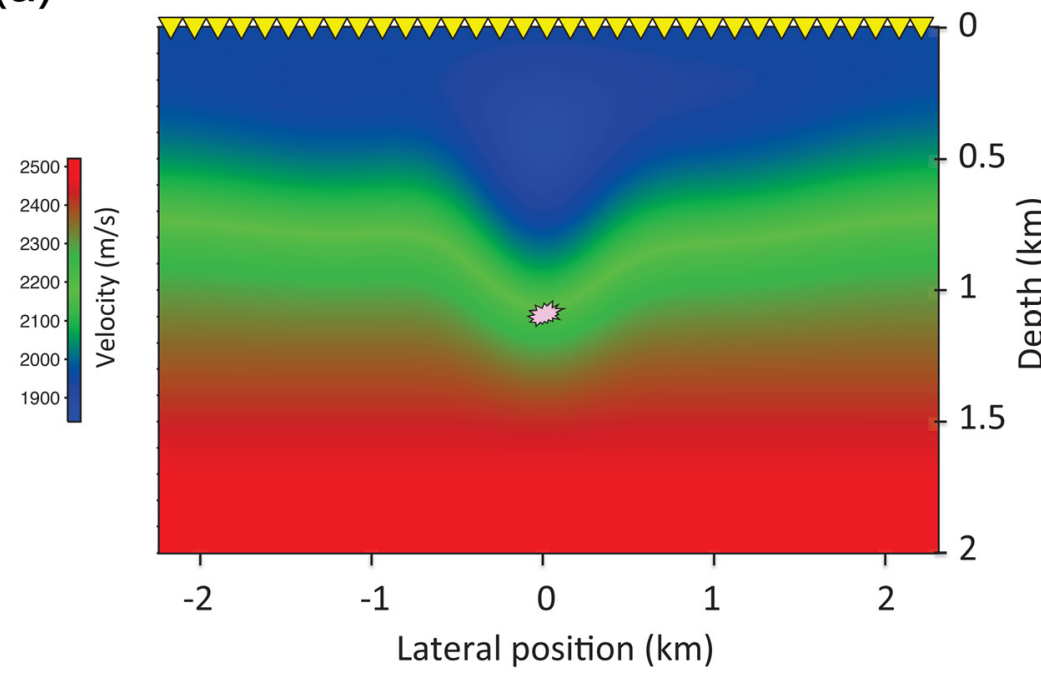

(b) Lateral position $(\mathrm{m})$

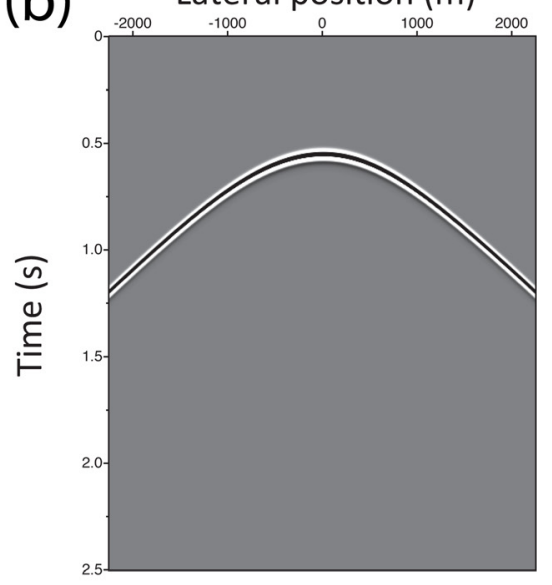

FIG. 8. (Color online) (a) Strongly smoothed version of the propagation velocity model of Fig. 1(a). (b) Direct arrival of the Green's function of the smoothed model, $G_{\mathrm{d}}\left(\mathbf{x}_{0}^{\prime \prime}, \mathbf{x}_{i}^{\prime}, t\right)$. 
iterations new events are generated, whereas during the higher iterations only the amplitudes of these events are modified. The second iteration [Fig. 6(c)] is already very close to the tenth iteration [Fig. 6(d)], which is taken as the final estimate of the focusing function $f_{2}\left(\mathbf{x}_{i}^{\prime}, \mathbf{x}_{0}^{\prime \prime}, t\right)$. Finally, step 4 involves constructing the pressure-normalized Green's function $G\left(\mathbf{x}_{0}^{\prime \prime}, \mathbf{x}_{i}^{\prime}, t\right)$ from the reflection response $R\left(\mathbf{x}_{0}^{\prime \prime}, \mathbf{x}_{0}, t\right)$ and the focusing function $f_{2}\left(\mathbf{x}_{i}^{\prime}, \mathbf{x}_{0}^{\prime \prime}, t\right)$. The result is represented by the thin black lines in Fig. 7(a) for fixed $\mathbf{x}_{i}^{\prime}$ and variable $\mathbf{x}_{0}^{\prime \prime}$. The thick gray lines represent the directly modeled Green's function. A time gain of $\exp (1.9 * t)$ has been applied to emphasize the multiple reflections. The central trace of Fig. 7(a) is shown with more detail in Fig. 7(b). Note that overall the match is very good. The triplications and internal multiples are very well recovered. Only the amplitudes of the steepest events at large horizontal source-receiver distance are underestimated. The cause for this is probably a combination of using a finite acquisition aperture and approximating $\mathcal{T}_{\mathrm{d}}^{\text {inv }}\left(\mathbf{x}_{i}^{\prime}, \mathbf{x}_{0}^{\prime \prime}, t\right)$ by $G_{\mathrm{d}}\left(\mathbf{x}_{0}^{\prime \prime}, \mathbf{x}_{i}^{\prime},-t\right)$.

In a next experiment, the direct arrival of the Green's function, $G_{\mathrm{d}}\left(\mathbf{x}_{0}^{\prime \prime}, \mathbf{x}_{i}^{\prime}, t\right)$, is modeled in a further smoothed version of the model of Fig. 1. Figure 8(a) shows the propagation velocity of the smoothed model and Fig. 8(b) shows the corresponding $G_{\mathrm{d}}\left(\mathbf{x}_{0}^{\prime \prime}, \mathbf{x}_{i}^{\prime}, t\right)$. Due to the smoothing, the triplication, which was still present in Fig. 1(e), has disappeared. Also the travel time at the apex is slightly smaller than that in Fig. 1(e). This estimate of the direct arrival [Fig. 8(b)] is

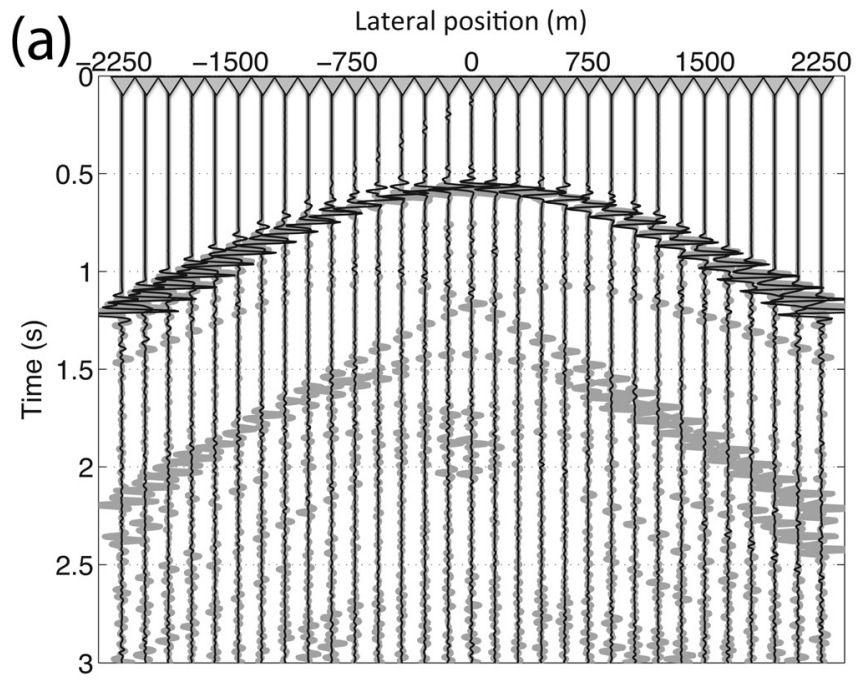

(b)

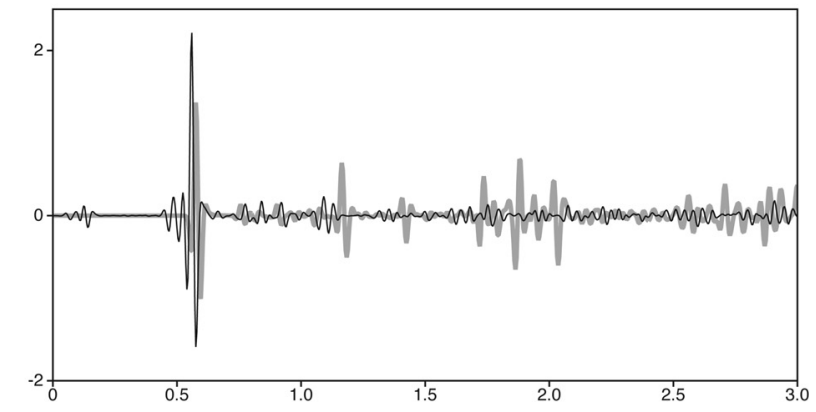

FIG. 9. Classical approach, as in Fig. 2, but this time using the direct arrivals in the strongly smoothed model of Fig. 8.

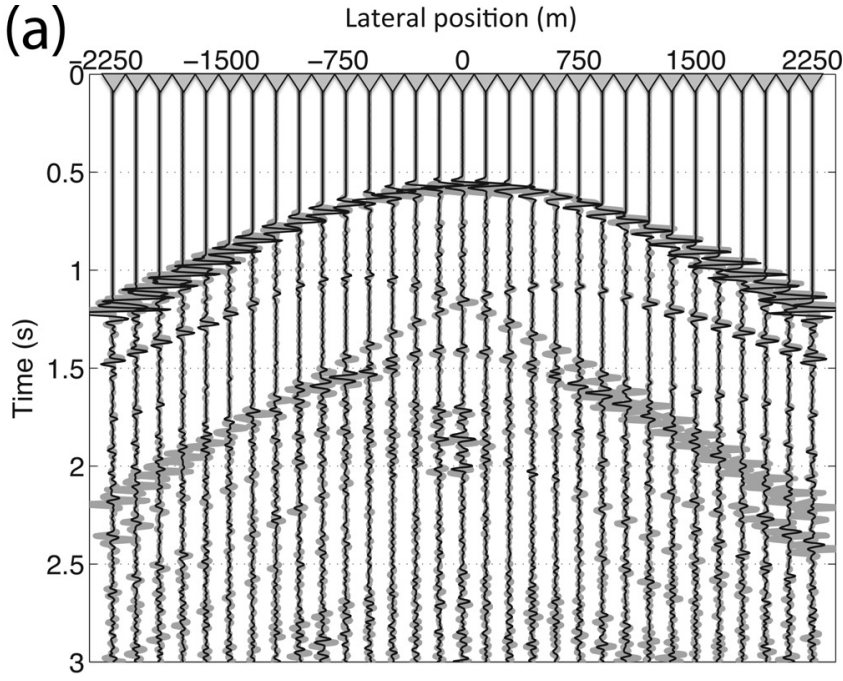

(b)

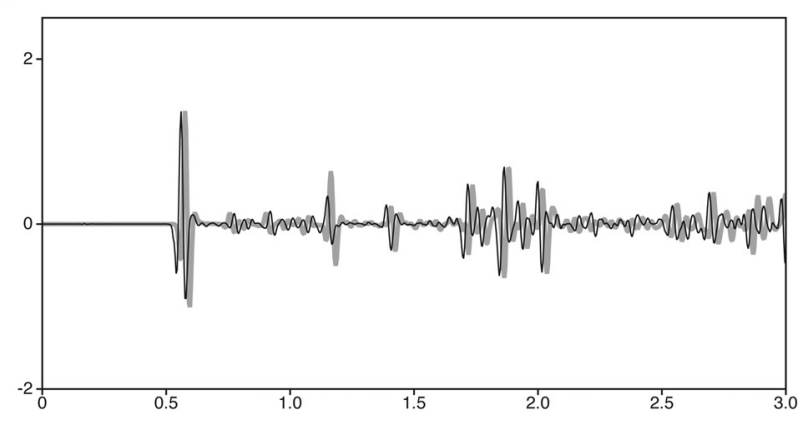

FIG. 10. Marchenko approach, as in Fig. 7, but this time using the direct arrivals in the strongly smoothed model of Fig. 8.

used, together with the reflection response $R\left(\mathbf{x}_{0}^{\prime \prime}, \mathbf{x}_{0}, t\right)$ of the original model [Fig. 1(b)], as input for the Green's function retrieval schemes. First the classical approach, as discussed in Sec. II, is applied. The result is shown in Fig. 9, in overlay with the reference Green's function. As expected, multiple reflections are not recovered. Next, the iterative Marchenko scheme is applied to the same data. The retrieved Green's function $G\left(\mathbf{x}_{0}^{\prime \prime}, \mathbf{x}_{i}^{\prime}, t\right)$ (again after ten iterations) is shown in Fig. 10. Amplitude errors and an overall small time shift are visible, but apart from that, the Marchenko scheme recovered the multiple reflections reasonably well.

These examples confirm that detailed knowledge of the medium is not required but that a smooth model suffices. The internal multiples in the retrieved Green's function $G\left(\mathbf{x}_{0}^{\prime \prime}, \mathbf{x}_{i}^{\prime}, t\right)$ come directly from the reflection response. Because the scatterers in the intervening medium do not need to be resolved in an intermediate step, the retrieval of internal multiples does not suffer from error accumulation. For comparison, full wave form inversion ${ }^{48,49}$ could start with the same smooth model, but the full Green's function could only be retrieved after the scatterers in the intervening medium were retrieved.

\section{CONCLUSIONS}

It has been shown that for a 3D inhomogeneous, lossless acoustic medium, the full Green's function between a virtual source inside the medium and receivers at the surface can be 
obtained from reflection measurements at the surface and an estimate of the direct arrival of the Green's function. No detailed information about the medium is required; a smooth background model that predicts the direct arrival suffices. ${ }^{50}$ The multiple reflections in the retrieved Green's function are extracted directly from the reflection measurements.

This method constitutes a significant step beyond seismic interferometry, because, unlike in seismic interferometry, no actual receiver is required at the position of the virtual source. The proposed method is based on a $3 \mathrm{D}$ extension of the singlesided Marchenko equation, which in this paper has been derived from reciprocity theorems for one-way wave fields. This 3D Marchenko equation formulates a relation between the single-sided reflection response and the scattering coda of a socalled focusing function. Once the scattering coda has been resolved, the Green's function can be constructed from the reflection response and the focusing function.

The proposed methodology has interesting applications for acoustic imaging. Because no receivers are needed at the virtual source positions, Green's functions can be obtained for virtual source positions throughout the medium. Because these Green's functions correctly contain all multiple scatterings, they can be used to obtain an accurate image of the medium, free of ghost images related to internal multiple scattering. ${ }^{20}$ This new form of imaging, also called 3D Marchenko imaging, uses the same information as standard reflection imaging, namely the reflection response at the surface and a background model of the propagation velocity (needed to evaluate the direct arrivals). Similar as in standard imaging, errors in the background model may cause mispositioning and defocusing. Nevertheless, the suppression of ghost images with Marchenko imaging is robust with respect to moderate errors in the background model. ${ }^{21}$

The proposed Green's function retrieval methodology and the resulting 3D Marchenko imaging methodology have interesting potential applications in ultrasonic, electromagnetic, and seismic imaging and monitoring at many scales. The main line of current research is directed toward extending the methodology beyond the scalar wave equation.

\section{APPENDIX: DERIVATION OF THE MARCHENKO EQUATION, USING PRESSURE-NORMALIZED ONE-WAY WAVE FIELDS}

The relation between acoustic pressure and fluxnormalized one-way wave fields is, according to Eq. (2), given by

$$
p=\mathcal{L}_{1}\left\{p^{+}+p^{-}\right\}
$$

Although flux-normalization facilitates the derivation of the 3D Marchenko equation, the actual implementation is complicated by the fact that pseudo-differential operators are involved. In this appendix the 3D Marchenko equation is derived, using pressure-normalized one-way wave fields. The relation between acoustic pressure and pressurenormalized one-way wave fields is very simple, namely,

$$
p=p^{+}+p^{-} \text {. }
$$

The derivation is, however, more complicated, as is seen below.

\section{Reciprocity theorems for pressure-normalized one-way wave fields}

The starting point for the derivation is formed by the reciprocity theorems of the convolution and correlation type for two-way wave fields, ${ }^{37,38}$ which, for a lossless medium between $\partial \mathbb{D}_{0}$ and $\partial \mathbb{D}_{i}$, read

$$
\begin{aligned}
\int_{\partial \mathbb{D}_{0}} \rho^{-1}\left\{\left(\partial_{3} p_{A}\right) p_{B}-p_{A}\left(\partial_{3} p_{B}\right)\right\} \mathrm{d} \mathbf{x}_{0} \\
\quad=\int_{\partial \mathbb{D}_{i}} \rho^{-1}\left\{\left(\partial_{3} p_{A}\right) p_{B}-p_{A}\left(\partial_{3} p_{B}\right)\right\} \mathrm{d} \mathbf{x}_{i}
\end{aligned}
$$

and

$$
\begin{aligned}
& \int_{\partial \mathbb{D}_{0}} \rho^{-1}\left\{\left(\partial_{3} p_{A}\right)^{*} p_{B}-p_{A}^{*}\left(\partial_{3} p_{B}\right)\right\} \mathrm{d} \mathbf{x}_{0} \\
& =\int_{\partial \mathbb{D}_{i}} \rho^{-1}\left\{\left(\partial_{3} p_{A}\right)^{*} p_{B}-p_{A}^{*}\left(\partial_{3} p_{B}\right)\right\} \mathrm{d} \mathbf{x}_{i}
\end{aligned}
$$

respectively. Substituting $p_{A}=p_{A}^{+}+p_{A}^{-}$and $p_{B}=p_{B}^{+}+p_{B}^{-}$, each product under the integrals is expanded into four products. Using one-way wave equations at $\partial \mathbb{D}_{0}$ and $\partial \mathbb{D}_{i}$, only the products containing oppositely propagating waves remain, whereas other terms cancel. ${ }^{26}$ The remaining terms are pairwise equal to each other, so they can be combined. This still leaves the choice to keep the terms with the operator $\partial_{3}$ acting on the fields in state $A$ or on those in state $B$. For the following derivations it appears to be useful to have $\partial_{3}$ acting on fields in state $B$ at $\partial \mathbb{D}_{0}$ and on fields in state $A$ at $\partial \mathbb{D}_{i}$. This gives

$$
\begin{aligned}
& -2 \int_{\partial \mathbb{D}_{0}} \rho^{-1}\left\{p_{A}^{+}\left(\partial_{3} p_{B}^{-}\right)+p_{A}^{-}\left(\partial_{3} p_{B}^{+}\right)\right\} \mathrm{d} \mathbf{x}_{0} \\
& =2 \int_{\partial \mathbb{D}_{i}} \rho^{-1}\left\{\left(\partial_{3} p_{A}^{+}\right) p_{B}^{-}+\left(\partial_{3} p_{A}^{-}\right) p_{B}^{+}\right\} \mathrm{d} \mathbf{x}_{i}
\end{aligned}
$$

and

$$
\begin{gathered}
-2 \int_{\partial \mathbb{D}_{0}} \rho^{-1}\left\{\left(p_{A}^{+}\right)^{*}\left(\partial_{3} p_{B}^{+}\right)+\left(p_{A}^{-}\right)^{*}\left(\partial_{3} p_{B}^{-}\right)\right\} \mathrm{d} \mathbf{x}_{0} \\
=2 \int_{\partial \mathbb{D}_{i}} \rho^{-1}\left\{\left(\partial_{3} p_{A}^{+}\right)^{*} p_{B}^{+}+\left(\partial_{3} p_{A}^{-}\right)^{*} p_{B}^{-}\right\} \mathrm{d} \mathbf{x}_{i}
\end{gathered}
$$

respectively. Note that in Eq. (A6) the evanescent wave fields at $\partial \mathbb{D}_{0}$ and $\partial \mathbb{D}_{i}$ are neglected. ${ }^{26}$

\section{Pressure-normalized one-way Green's functions and focusing functions}

The Green's function $G\left(\mathbf{x}, \mathbf{x}_{0}^{\prime \prime}, t\right)$ is defined as the solution of the scalar wave equation in the actual medium, with a source at $\mathbf{x}_{0}^{\prime \prime}$, according to $\rho \nabla \cdot((1 / \rho) \nabla G)-\left(1 / c^{2}\right)\left(\partial^{2} G /\right.$ $\left.\partial t^{2}\right)=-\rho \delta\left(\mathbf{x}-\mathbf{x}_{0}^{\prime \prime}\right)(\partial \delta(t) / \partial t)$. Defined in this way, the Green's function is the response to an impulsive point source of volume injection rate at $\mathbf{x}_{0}^{\prime \prime} .{ }^{51}$ In the frequency domain $G\left(\mathbf{x}, \mathbf{x}_{0}^{\prime \prime}, \omega\right)$ obeys $\rho \nabla \cdot((1 / \rho) \nabla G)+\left(\omega^{2} / c^{2}\right) G=-j \omega \rho \delta$ 
$\times\left(\mathbf{x}-\mathbf{x}_{0}^{\prime \prime}\right)$. In the following, $\mathbf{x}_{0}^{\prime \prime}$ is chosen again just above $\partial \mathbb{D}_{0}$, see Fig. 4. At and below $\partial \mathbb{D}_{0}$ the Green's function consists of a down-going part $G^{+, q}\left(\mathbf{x}, \mathbf{x}_{0}^{\prime \prime}, \omega\right)$ and an up-going part $G^{-, q}\left(\mathbf{x}, \mathbf{x}_{0}^{\prime \prime}, \omega\right)$, coupled by the inhomogeneities of the medium below $\partial \mathbb{D}_{0}$ (recall that the medium above $\partial \mathbb{D}_{0}$ is homogeneous). Unlike the flux-normalized one-way Green's functions introduced in Sec. V, the pressure-normalized oneway Green's functions are decomposed only at the observation point $\mathbf{x}$, which is denoted by the first superscript ( + or $-)$; the second superscript $(q)$ refers to the volume-injection source at $\mathbf{x}_{0}^{\prime \prime}$. In agreement with Eq. (A2) the two-way Green's function is related to the pressure-normalized one-way Green's functions, according to

$$
\begin{aligned}
G\left(\mathbf{x}, \mathbf{x}_{0}^{\prime \prime}, \omega\right) & =G^{p, q}\left(\mathbf{x}, \mathbf{x}_{0}^{\prime \prime}, \omega\right) \\
& =G^{+, q}\left(\mathbf{x}, \mathbf{x}_{0}^{\prime \prime}, \omega\right)+G^{-, q}\left(\mathbf{x}, \mathbf{x}_{0}^{\prime \prime}, \omega\right) .
\end{aligned}
$$

With these definitions, the pressure-normalized version of Eq. (16) is

$$
\left.\partial_{3} G^{+, q}\left(\mathbf{x}, \mathbf{x}_{0}^{\prime \prime}, \omega\right)\right|_{x_{3}=x_{3,0}}=-\frac{1}{2} j \omega \rho\left(\mathbf{x}_{0}^{\prime \prime}\right) \delta\left(\mathbf{x}_{\mathrm{H}}-\mathbf{x}_{\mathrm{H}}^{\prime \prime}\right) .
$$

To derive the pressure-normalized version of Eq. (17), substitute $p_{B}^{ \pm}(\mathbf{x}, \omega)=G^{ \pm, q}\left(\mathbf{x}, \mathbf{x}_{0}^{\prime \prime}, \omega\right)$ into Eq. (A5). Moreover, replace $\partial \mathbb{D}_{i}$ by $\partial \mathbb{D}_{m}$, where $\partial \mathbb{D}_{m}$ is a boundary which lies below all inhomogeneities, so that $p_{A}^{-}\left(\mathbf{x}_{m}, \omega\right)=p_{B}^{-}\left(\mathbf{x}_{m}, \omega\right)$ $=0$. This gives

$$
p_{A}^{-}\left(\mathbf{x}_{0}^{\prime \prime}, \omega\right)=\int_{\partial \mathbb{D}_{0}}\left(\frac{\left.\partial_{3} G^{-, q}\left(\mathbf{x}, \mathbf{x}_{0}^{\prime \prime}, \omega\right)\right|_{x_{3}=x_{3,0}}}{\frac{1}{2} j \omega \rho\left(\mathbf{x}_{0}\right)}\right) p_{A}^{+}\left(\mathbf{x}_{0}, \omega\right) \mathrm{d} \mathbf{x}_{0} .
$$

Introducing the pressure-normalized reflection response $R\left(\mathbf{x}_{0}^{\prime \prime}, \mathbf{x}_{0}, \omega\right)$ via

$$
p_{A}^{-}\left(\mathbf{x}_{0}^{\prime \prime}, \omega\right)=\int_{\partial \mathbb{D}_{0}} R\left(\mathbf{x}_{0}^{\prime \prime}, \mathbf{x}_{0}, \omega\right) p_{A}^{+}\left(\mathbf{x}_{0}, \omega\right) \mathrm{d} \mathbf{x}_{0},
$$

it follows that

$$
\partial_{3} G^{-, q}\left(\mathbf{x}, \mathbf{x}_{0}^{\prime \prime}, \omega\right)_{x_{3}=x_{3,0}}=\frac{1}{2} j \omega \rho\left(\mathbf{x}_{0}\right) R\left(\mathbf{x}_{0}^{\prime \prime}, \mathbf{x}_{0}, \omega\right),
$$

which is the pressure-normalized version of Eq. (17).

The focusing functions are again defined in the reference configuration of Fig. 3. Analogous to Eq. (A8), the pressure-normalized versions of Eqs. (9) and (11) are

$$
\left.\partial_{3} f_{1}^{+}\left(\mathbf{x}, \mathbf{x}_{i}^{\prime}, \omega\right)\right|_{x_{3}=x_{3, i}}=-\frac{1}{2} j \omega \rho\left(\mathbf{x}_{i}^{\prime}\right) \delta\left(\mathbf{x}_{\mathrm{H}}-\mathbf{x}_{\mathrm{H}}^{\prime}\right)
$$

and

$$
\left.\partial_{3} f_{2}^{-}\left(\mathbf{x}, \mathbf{x}_{0}^{\prime \prime}, \omega\right)\right|_{x_{3}=x_{3,0}}=\frac{1}{2} j \omega \rho\left(\mathbf{x}_{0}^{\prime \prime}\right) \delta\left(\mathbf{x}_{\mathrm{H}}-\mathbf{x}_{\mathrm{H}}^{\prime \prime}\right),
$$

respectively. Substituting $p_{A}^{ \pm}(\mathbf{x}, \omega)=f_{1}^{ \pm}\left(\mathbf{x}, \mathbf{x}_{i}^{\prime}, \omega\right), \quad p_{B}^{ \pm}(\mathbf{x}, \omega)$ $=f_{2}^{ \pm}\left(\mathbf{x}, \mathbf{x}_{0}^{\prime \prime}, \omega\right)$, and $\left.\partial_{3} p_{A}^{-}(\mathbf{x}, \omega)\right|_{x_{3}=x_{3, i}}=\left.\partial_{3} p_{B}^{+}(\mathbf{x}, \omega)\right|_{x_{3}=x_{3,0}}$ $=0$ into Eqs. (A5) and (A6), using Eqs. (A12) and (A13), yields

$$
f_{1}^{+}\left(\mathbf{x}_{0}^{\prime \prime}, \mathbf{x}_{i}^{\prime}, \omega\right)=f_{2}^{-}\left(\mathbf{x}_{i}^{\prime}, \mathbf{x}_{0}^{\prime \prime}, \omega\right)
$$

and

$$
-\left\{f_{1}^{-}\left(\mathbf{x}_{0}^{\prime \prime}, \mathbf{x}_{i}^{\prime}, \omega\right)\right\}^{*}=f_{2}^{+}\left(\mathbf{x}_{i}^{\prime}, \mathbf{x}_{0}^{\prime \prime}, \omega\right),
$$

respectively.

Substituting $p_{A}^{ \pm}(\mathbf{x}, \omega)=f_{1}^{ \pm}\left(\mathbf{x}, \mathbf{x}_{i}^{\prime}, \omega\right), p_{B}^{ \pm}(\mathbf{x}, \omega)=G^{ \pm, q}(\mathbf{x}$, $\left.\mathbf{x}_{0}^{\prime \prime}, \omega\right)$, and $\left.\partial_{3} p_{A}^{-}(\mathbf{x}, \omega)\right|_{x_{3}=x_{3, i}}=0$ into Eqs. (A5) and (A6), using Eqs. (A8) and (A11)-(A13), yields

$$
\begin{aligned}
& \int_{\partial \mathbb{D}_{0}} f_{1}^{+}\left(\mathbf{x}_{0}, \mathbf{x}_{i}^{\prime}, \omega\right) R\left(\mathbf{x}_{0}^{\prime \prime}, \mathbf{x}_{0}, \omega\right) \mathrm{d} \mathbf{x}_{0}-f_{1}^{-}\left(\mathbf{x}_{0}^{\prime \prime}, \mathbf{x}_{i}^{\prime}, \omega\right) \\
& \quad=G^{-, q}\left(\mathbf{x}_{i}^{\prime}, \mathbf{x}_{0}^{\prime \prime}, \omega\right)
\end{aligned}
$$

and

$$
\begin{aligned}
& -\int_{\partial \mathbb{D}_{0}}\left\{f_{1}^{-}\left(\mathbf{x}_{0}, \mathbf{x}_{i}^{\prime}, \omega\right)\right\}^{*} R\left(\mathbf{x}_{0}^{\prime \prime}, \mathbf{x}_{0}, \omega\right) \mathrm{d} \mathbf{x}_{0}+\left\{f_{1}^{+}\left(\mathbf{x}_{0}^{\prime \prime}, \mathbf{x}_{i}^{\prime}, \omega\right)\right\}^{*} \\
& =G^{+, q}\left(\mathbf{x}_{i}^{\prime}, \mathbf{x}_{0}^{\prime \prime}, \omega\right)
\end{aligned}
$$

respectively. Adding these expressions, using Eqs. (A14) and (A15), and bringing the Green's functions to the lefthand side, gives

$$
\begin{aligned}
G^{+, q} & \left(\mathbf{x}_{i}^{\prime}, \mathbf{x}_{0}^{\prime \prime}, \omega\right)+G^{-, q}\left(\mathbf{x}_{i}^{\prime}, \mathbf{x}_{0}^{\prime \prime}, \omega\right) \\
\quad= & \int_{\partial \mathbb{D}_{0}} f_{2}\left(\mathbf{x}_{i}^{\prime}, \mathbf{x}_{0}, \omega\right) R\left(\mathbf{x}_{0}^{\prime \prime}, \mathbf{x}_{0}, \omega\right) \mathrm{d} \mathbf{x}_{0}+\left\{f_{2}\left(\mathbf{x}_{i}^{\prime}, \mathbf{x}_{0}^{\prime \prime}, \omega\right)\right\}^{*}
\end{aligned}
$$

Note that, despite the different normalization, Eqs. (A16)-(A18) are identical to Eqs. (18)-(20). Keep in mind that, unlike in Eqs. (18)-(20), $R\left(\mathbf{x}_{0}^{\prime \prime}, \mathbf{x}_{0}, \omega\right)$ in Eqs. (A16)-(A18) does not obey source-receiver reciprocity. Furthermore note that because the one-way Green's functions on the left-hand sides of Eqs. (A16)-(A18) are pressure-normalized, the two-way Green's function is simply given by

$$
G\left(\mathbf{x}_{i}^{\prime}, \mathbf{x}_{0}^{\prime \prime}, \omega\right)=G^{+, q}\left(\mathbf{x}_{i}^{\prime}, \mathbf{x}_{0}^{\prime \prime}, \omega\right)+G^{-, q}\left(\mathbf{x}_{i}^{\prime}, \mathbf{x}_{0}^{\prime \prime}, \omega\right)
$$

which is the pressure-normalized version of Eq. (21).

\section{Pressure-normalized Marchenko equation}

The derivation of the pressure-normalized 3D Marchenko equation from Eq. (A18) is very similar to the derivation in Sec. VI of the flux-normalized 3D Marchenko equation from Eq. (20). The main difference lies in the ansatz that is made for $f_{2}\left(\mathbf{x}_{i}, \mathbf{x}_{0}^{\prime \prime}, t\right)$. Again, first an auxiliary property of $f_{2}^{-}\left(\mathbf{x}_{i}, \mathbf{x}_{0}^{\prime \prime}, t\right)$ is derived. Let $T\left(\mathbf{x}_{i}, \mathbf{x}_{0}^{\prime \prime}, t\right)$ in Fig. 5 denote the pressure-normalized transmission response of the reference configuration, with $\mathbf{x}_{0}^{\prime \prime}$ just above $\partial \mathbb{D}_{0}$. Substituting $p_{A}^{+}\left(\mathbf{x}_{0}, \omega\right)=\delta\left(\mathbf{x}_{H}-\mathbf{x}_{\mathrm{H}}^{\prime \prime}\right), p_{A}^{+}\left(\mathbf{x}_{i}, \omega\right)=T\left(\mathbf{x}_{i}, \mathbf{x}_{0}^{\prime \prime}, \omega\right)$, $p_{A}^{-}\left(\mathbf{x}_{i}, \omega\right)=0$, and $p_{B}^{ \pm}(\mathbf{x}, \omega)=f_{2}^{ \pm}\left(\mathbf{x}, \mathbf{x}_{0}^{\prime}, \omega\right)$ into Eq. (A5), using Eq. (A13) (with single instead of double primes), yields 


$$
\delta\left(\mathbf{x}_{\mathrm{H}}^{\prime}-\mathbf{x}_{\mathrm{H}}^{\prime \prime}\right)=\int_{\partial \mathbb{D}_{i}} \mathcal{T}\left(\mathbf{x}_{i}, \mathbf{x}_{0}^{\prime \prime}, \omega\right) f_{2}^{-}\left(\mathbf{x}_{i}, \mathbf{x}_{0}^{\prime}, \omega\right) \mathrm{d} \mathbf{x}_{i},
$$

where the modified transmission response $\mathcal{T}\left(\mathbf{x}_{i}, \mathbf{x}_{0}^{\prime \prime}, \omega\right)$ is given by

$$
\mathcal{T}\left(\mathbf{x}_{i}, \mathbf{x}_{0}^{\prime \prime}, \omega\right)=\frac{\left.\partial_{3} T\left(\mathbf{x}, \mathbf{x}_{0}^{\prime \prime}, \omega\right)\right|_{x_{3}=x_{3, i}}}{-\frac{1}{2} j \omega \rho\left(\mathbf{x}_{i}\right)}
$$

Hence, $f_{2}^{-}\left(\mathbf{x}_{i}, \mathbf{x}_{0}^{\prime \prime}, \omega\right)$ is the inverse of the modified transmission response $\mathcal{T}\left(\mathbf{x}_{i}, \mathbf{x}_{0}^{\prime \prime}, \omega\right)$ in the sense of Eq. (A20). In the time domain this is formulated as

$$
f_{2}^{-}\left(\mathbf{x}_{i}, \mathbf{x}_{0}^{\prime \prime}, t\right)=\mathcal{T}^{\mathrm{inv}}\left(\mathbf{x}_{i}, \mathbf{x}_{0}^{\prime \prime}, t\right) .
$$

Following the same reasoning as in Sec. VI, the ansatz for the pressure-normalized version of $f_{2}\left(\mathbf{x}_{i}, \mathbf{x}_{0}^{\prime \prime}, t\right)$ is thus written as

$$
f_{2}\left(\mathbf{x}_{i}, \mathbf{x}_{0}^{\prime \prime}, t\right)=\mathcal{T}_{\mathrm{d}}^{\mathrm{inv}}\left(\mathbf{x}_{i}, \mathbf{x}_{0}^{\prime \prime}, t\right)+M\left(\mathbf{x}_{i}, \mathbf{x}_{0}^{\prime \prime}, t\right),
$$

where $\mathcal{T}_{\mathrm{d}}^{\text {inv }}\left(\mathbf{x}_{i}, \mathbf{x}_{0}^{\prime \prime}, t\right)$ is the inverse of the direct arrival of the modified transmission response,

$$
\mathcal{T}_{\mathrm{d}}\left(\mathbf{x}_{i}, \mathbf{x}_{0}^{\prime \prime}, \omega\right)=\frac{\left.\partial_{3} T_{\mathrm{d}}\left(\mathbf{x}, \mathbf{x}_{0}^{\prime \prime}, \omega\right)\right|_{x_{3}=x_{3, i}}}{-\frac{1}{2} j \omega \rho\left(\mathbf{x}_{i}\right)}
$$

and where $M\left(\mathbf{x}_{i}, \mathbf{x}_{0}^{\prime \prime}, t\right)$ is the pressure-normalized coda following the direct arrival, with

$$
M\left(\mathbf{x}_{i}, \mathbf{x}_{0}^{\prime \prime}, t\right)=0, \quad \text { for } \quad t \leq-t_{d}\left(\mathbf{x}_{i}, \mathbf{x}_{0}^{\prime \prime}\right)
$$

Note that the direct arrival of the pressure-normalized transmission response in Eq. (A24), $T_{\mathrm{d}}\left(\mathbf{x}_{i}, \mathbf{x}_{0}^{\prime \prime}, \omega\right)$, is related to the direct arrival of the Green's function, $G_{\mathrm{d}}\left(\mathbf{x}_{i}, \mathbf{x}_{0}^{\prime \prime}, \omega\right)=G_{\mathrm{d}}\left(\mathbf{x}_{0}^{\prime \prime}\right.$, $\mathbf{x}_{i}, \omega$ ), analogous to Eq. (A11), via

$$
T_{\mathrm{d}}\left(\mathbf{x}_{i}, \mathbf{x}_{0}^{\prime \prime}, \omega\right)=\frac{\left.\partial_{3} G_{\mathrm{d}}\left(\mathbf{x}^{\prime \prime}, \mathbf{x}_{i}, \omega\right)\right|_{x_{3}^{\prime \prime}=x_{3,0}}}{\frac{1}{2} j \omega \rho\left(\mathbf{x}_{0}^{\prime \prime}\right)} .
$$

The pressure-normalized 3D Marchenko equation is obtained by transforming Eq. (A18) to the time domain, substituting Eq. (A23), and evaluating the result only for $t<t_{\mathrm{d}}\left(\mathbf{x}_{i}^{\prime}, \mathbf{x}_{0}^{\prime \prime}\right)$. It is solved for $M\left(\mathbf{x}_{i}^{\prime}, \mathbf{x}_{0}^{\prime \prime}, t\right)$ by the following iterative scheme:

$$
\begin{aligned}
M_{k}\left(\mathbf{x}_{i}^{\prime}, \mathbf{x}_{0}^{\prime \prime},-t\right)= & M_{0}\left(\mathbf{x}_{i}^{\prime}, \mathbf{x}_{0}^{\prime \prime},-t\right) \\
& -\int_{\partial \mathbb{D}_{0}} \mathrm{~d} \mathbf{x}_{0} \int_{-t_{\mathrm{d}}^{\epsilon}\left(\mathbf{x}_{i}^{\prime}, \mathbf{x}_{0}\right)}^{t} M_{k-1}\left(\mathbf{x}_{i}^{\prime}, \mathbf{x}_{0}, t^{\prime}\right) \\
& \times R\left(\mathbf{x}_{0}^{\prime \prime}, \mathbf{x}_{0}, t-t^{\prime}\right) \mathrm{d} t^{\prime},
\end{aligned}
$$

with

$$
\begin{aligned}
& M_{0}\left(\mathbf{x}_{i}^{\prime}, \mathbf{x}_{0}^{\prime \prime},-t\right) \\
& \quad=-\int_{\partial \mathbb{D}_{0}} \mathrm{~d} \mathbf{x}_{0} \int_{-\infty}^{-t_{\mathrm{d}}^{\epsilon}\left(\mathbf{x}_{i}^{\prime}, \mathbf{x}_{0}\right)} \mathcal{T}_{\mathrm{d}}^{\text {inv }}\left(\mathbf{x}_{i}^{\prime}, \mathbf{x}_{0}, t^{\prime}\right) R\left(\mathbf{x}_{0}^{\prime \prime}, \mathbf{x}_{0}, t-t^{\prime}\right) \mathrm{d} t^{\prime},
\end{aligned}
$$

for $t<t_{\mathrm{d}}\left(\mathbf{x}_{i}^{\prime}, \mathbf{x}_{0}^{\prime \prime}\right)$, and

$$
M_{k}\left(\mathbf{x}_{i}^{\prime}, \mathbf{x}_{0}^{\prime \prime},-t\right)=0, \quad \text { for } \quad t \geq t_{d}\left(\mathbf{x}_{i}^{\prime}, \mathbf{x}_{0}^{\prime \prime}\right) .
$$

Using Eq. (A23), the successive iterations for the focusing function $f_{2}\left(\mathbf{x}_{i}^{\prime}, \mathbf{x}_{0}^{\prime \prime}, t\right)$ read

$$
\begin{aligned}
& f_{2,0}\left(\mathbf{x}_{i}^{\prime}, \mathbf{x}_{0}^{\prime \prime}, t\right)=\mathcal{T}_{\mathrm{d}}^{\mathrm{inv}}\left(\mathbf{x}_{i}^{\prime}, \mathbf{x}_{0}^{\prime \prime}, t\right), \\
& f_{2, k}\left(\mathbf{x}_{i}^{\prime}, \mathbf{x}_{0}^{\prime \prime}, t\right)=\mathcal{T}_{\mathrm{d}}^{\mathrm{inv}}\left(\mathbf{x}_{i}^{\prime}, \mathbf{x}_{0}^{\prime \prime}, t\right)+M_{k-1}\left(\mathbf{x}_{i}^{\prime}, \mathbf{x}_{0}^{\prime \prime}, t\right) .
\end{aligned}
$$

In its simplest form, $\mathcal{T}_{\mathrm{d}}^{\text {inv }}\left(\mathbf{x}_{i}^{\prime}, \mathbf{x}_{0}^{\prime \prime}, t\right)$ is approximated by $G_{\mathrm{d}}\left(\mathbf{x}_{i}^{\prime}\right.$, $\left.\mathbf{x}_{0}^{\prime \prime},-t\right)$. This approximation properly accounts for travel times and geometrical spreading, but ignores the effect of transmission losses at the interfaces. ${ }^{42}$

${ }^{1}$ G. L. Lamb, Elements of Soliton Theory (John Wiley and Sons, Inc., New York, 1980), pp. 46-67.

${ }^{2}$ R. Burridge, "The Gelfand-Levitan, the Marchenko, and the GopinathSondhi integral equations of inverse scattering theory, regarded in the context of inverse impulse-response problems," Wave Motion 2, 305-323 (1980).

${ }^{3}$ D. B. Ge, "An iterative technique in one-dimensional profile inversion," Inverse Probl. 3, 399-406 (1987).

${ }^{4} \mathrm{~K}$. Chadan and P. C. Sabatier, Inverse Problems in Quantum Scattering Theory (Springer, Berlin, 1989), Chaps. 5 and 7.

${ }^{5}$ J. H. Rose, " 'Single-sided' focusing of the time-dependent Schrödinger equation,” Phys. Rev. A 65, 012707 (2001).

${ }^{6}$ J. H. Rose, "Time reversal, focusing and exact inverse scattering," in Imaging of Complex Media with Acoustic and Seismic Waves, edited by M. Fink, W. A. Kuperman, J. P. Montagner, and A. Tourin (Springer, Berlin, 2002), pp. 97-106.

${ }^{7}$ J. H. Rose, "'Single-sided' autofocusing of sound in layered materials," Inverse Probl. 18, 1923-1934 (2002).

${ }^{8}$ F. Broggini and R. Snieder, "Connection of scattering principles: A visual and mathematical tour," Eur. J. Phys. 33, 593-613 (2012).

${ }^{9}$ R. L. Weaver and O. I. Lobkis, "Ultrasonics without a source: Thermal fluctuation correlations at MHz frequencies," Phys. Rev. Lett. 87, 134301 (2001).

${ }^{10} \mathrm{M}$. Campillo and A. Paul, "Long-range correlations in the diffuse seismic coda," Science 299, 547-549 (2003).

${ }^{11}$ K. G. Sabra, P. Gerstoft, P. Roux, W. A. Kuperman, and M. C. Fehler, "Surface wave tomography from microseisms in Southern California," Geophys. Res. Lett. 32, L14311, doi:10.1029/2005GL023155 (2005).

${ }^{12}$ D. Draganov, K. Wapenaar, W. Mulder, J. Singer, and A. Verdel, "Retrieval of reflections from seismic background-noise measurements," Geophys. Res. Lett. 34, L04305, doi:10.1029/2006GL028735 (2007).

${ }^{13}$ G. T. Schuster, "Theory of daylight/interferometric imaging: Tutorial," in EAGE, Extended Abstracts, A032 (2001).

${ }^{14}$ G. T. Schuster, Seismic Interferometry (Cambridge University Press, Cambridge, 2009), Chaps. 3-8.

${ }^{15} \mathrm{~A}$. Bakulin and R. Calvert, "The virtual source method: Theory and case study," Geophysics 71, SI139-SI150 (2006).

${ }^{16}$ A. Curtis, P. Gerstoft, H. Sato, R. Snieder, and K. Wapenaar, "Seismic interferometry-Turning noise into signal," The Leading Edge 25, 1082-1092 (2006).

${ }^{17}$ F. Broggini, R. Snieder, and K. Wapenaar, "Focusing the wavefield inside an unknown 1D medium: Beyond seismic interferometry," Geophysics 77, A25-A28 (2012).

${ }^{18}$ R. G. Newton, "Inverse scattering. IV. Three dimensions: Generalized Marchenko construction with bound states, and generalized Gel'fandLevitan equations," J. Math. Phys. 23, 594-604 (1982).

${ }^{19}$ D. E. Budreck and J. H. Rose, "Three-dimensional inverse scattering in anisotropic elastic media," Inverse Probl. 6, 331-348 (1990).

${ }^{20} \mathrm{~K}$. Wapenaar, J. Thorbecke, J. van der Neut, F. Broggini, E. Slob, and R. Snieder, "Marchenko imaging," Geophysics 79 (2014) (to be published).

${ }^{21}$ F. Broggini, R. Snieder, and K. Wapenaar, "Data-driven wave field focusing and imaging with multidimensional deconvolution: Numerical examples for reflection data with internal multiples," Geophysics 79 (2014) (to be published). 
${ }^{22}$ J. R. Berryhill, "Wave-equation datuming before stack," Geophysics 49, 2064-2066 (1984).

${ }^{23}$ A. J. Berkhout and C. P. A. Wapenaar, "A unified approach to acoustical reflection imaging. Part II: The inverse problem,” J. Acoust. Soc. Am. 93, 2017-2023 (1993).

${ }^{24}$ J. P. Corones, M. E. Davison, and R. J. Krueger, "Direct and inverse scattering in the time domain via invariant imbedding equations," J. Acoust. Soc. Am. 74, 1535-1541 (1983).

${ }^{25}$ L. Fishman, J. J. McCoy, and S. C. Wales, "Factorization and path integration of the Helmholtz equation: Numerical algorithms," J. Acoust. Soc. Am. 81, 1355-1376 (1987).

${ }^{26}$ C. P. A. Wapenaar and A. J. Berkhout, Elastic Wave Field Extrapolation (Elsevier, Amsterdam, 1989), Chaps. 3 and 5, App. B.

${ }^{27}$ L. Fishman, "One-way propagation methods in direct and inverse scalar wave propagation modeling," Radio Sci. 28, 865-876, doi:10.1029/93RS01632 (1993).

${ }^{28}$ M. V. de Hoop, "Directional decomposition of transient acoustic wave fields," Ph.D. thesis, Delft University of Technology (1992), Chap. 5.

${ }^{29}$ M. V. de Hoop, "Generalization of the Bremmer coupling series," J. Math. Phys. 37, 3246-3282 (1996).

${ }^{30}$ A. J. Haines and M. V. de Hoop, "An invariant imbedding analysis of general wave scattering problems," J. Math. Phys. 37, 3854-3881 (1996).

${ }^{31}$ C. P. A. Wapenaar, M. W. P. Dillen, and J. T. Fokkema, "Reciprocity theorems for electromagnetic or acoustic one-way wave fields in dissipative inhomogeneous media," Radio Sci. 36, 851-863, doi:10.1029/2000RS002394 (2001).

${ }^{32}$ A. J. Haines, "Multi-source, multi-receiver synthetic seismograms for laterally heterogeneous media using F-K domain propagators," Geophys. J. Int. 95, 237-260 (1988)

${ }^{33}$ B. L. N. Kennett, K. Koketsu, and A. J. Haines, "Propagation invariants, reflection and transmission in anisotropic, laterally heterogeneous media," Geophys. J. Int. 103, 95-101 (1990).

${ }^{34}$ K. Koketsu, B. L. N. Kennett, and H. Takenaka, “2-D reflectivity method and synthetic seismograms for irregularly layered structures-II. Invariant embedding approach," Geophys. J. Int. 105, 119-130 (1991).

${ }^{35}$ H. Takenaka, B. L. N. Kennett, and K. Koketsu, "The integral operator representation of propagation invariants for elastic waves in irregularly layered media," Wave Motion 17, 299-317 (1993).

${ }^{36}$ C. P. A. Wapenaar, "One-way representations of seismic data," Geophys. J. Int. 127, 178-188 (1996).
${ }^{37}$ A. T. de Hoop, "Time-domain reciprocity theorems for acoustic wave fields in fluids with relaxation," J. Acoust. Soc. Am. 84, 1877-1882 (1988).

${ }^{38}$ J. T. Fokkema and P. M. van den Berg, Seismic Applications of Acoustic Reciprocity (Elsevier, Amsterdam, 1993), Chaps. 5 and 6.

${ }^{39} \mathrm{~K}$. Wapenaar, J. Thorbecke, and D. Draganov, "Relations between reflection and transmission responses of three-dimensional inhomogeneous media," Geophys. J. Int. 156, 179-194 (2004).

${ }^{40} \mathrm{~K}$. Wapenaar, F. Broggini, E. Slob, and R. Snieder, "Three-dimensional single-sided Marchenko inverse scattering, data-driven focusing, Green's function retrieval, and their mutual relations," Phys. Rev. Lett. 110, 084301 (2013).

${ }^{41}$ E. Slob, K. Wapenaar, F. Broggini, and R. Snieder, "Seismic reflector imaging using internal multiples with Marchenko-type equations" Geophysics 79(2), S63-S76 (2014).

${ }^{42}$ C. P. A. Wapenaar, G. L. Peels, V. Budejicky, and A. J. Berkhout, "Inverse extrapolation of primary seismic waves," Geophysics 54, 853-863 (1989).

${ }^{43}$ C. Prada, F. Wu, and M. Fink, "The iterative time reversal mirror: A solution to self-focusing in the pulse echo mode," J. Acoust. Soc. Am. 90, 1119-1129 (1991).

${ }^{44}$ G. Montaldo, J. F. Aubry, M. Tanter, and M. Fink, "Spatio-temporal coding in complex media for optimum beamforming: The iterative timereversal approach" IEEE Trans. Ultrason. Ferroelectr., Freq. Control 52, 220-230 (2005).

${ }^{45}$ M. Fink, "Time-reversal acoustics," J. Phys. Conf. Ser. 118, 012001 (2008).

${ }^{46}$ D. J. Verschuur, A. J. Berkhout, and C. P. A. Wapenaar, "Adaptive surface-related multiple elimination," Geophysics 57, 1166-1177 (1992).

${ }^{47}$ G. J. A. van Groenestijn and D. J. Verschuur, "Estimation of primaries and near-offset reconstruction by sparse inversion: Marine data applications," Geophysics 74, R119-R128 (2009).

${ }^{48}$ J. Virieux and S. Operto, "An overview of full-waveform inversion in exploration geophysics," Geophysics 74, WCC1-WCC26 (2009).

${ }^{49}$ Q. Liu and Y. J. Gu, "Seismic imaging: From classical to adjoint tomography," Tectonophysics 566-567, 31-66 (2012).

${ }^{50}$ F. Broggini, R. Snieder, and K. Wapenaar, "Focusing inside an unknown medium using reflection data with internal multiples: numerical examples for a laterally-varying velocity model, spatially-extended virtual source, and inaccurate direct arrivals," in SEG, Expanded Abstracts, SPMUL 1.8 (2012).

${ }^{51}$ A. T. de Hoop, Handbook of Radiation and Scattering of Waves (Academic Press, London, 1995), Chap. 2. 